\title{
Extended complete Tchebycheffian spline spaces over planar T-meshes: Dimension bounds and dimension instabilities
}

\author{
Cesare Bracco $^{\mathrm{a}}$, Tom Lyche ${ }^{\mathrm{b}}$, Carla Manni ${ }^{\mathrm{c}}$, Hendrik Speleers ${ }^{\mathrm{c}}$ \\ ${ }^{a}$ Department of Mathematics and Computer Science, University of Florence, Italy \\ ${ }^{b}$ Department of Mathematics, University of Oslo, Norway \\ ${ }^{c}$ Department of Mathematics, University of Rome "Tor Vergata", Italy
}

\begin{abstract}
We consider extended complete Tchebycheffian spline spaces over planar T-meshes and we study their dimension. We show that the structure of extended complete Tchebycheff spaces allows us to fully generalize the dimension upper bounds known in the literature for polynomial spline spaces over Tmeshes. Moreover, we illustrate that the dimension of extended complete Tchebycheffian spline spaces over T-meshes can be unstable for certain configurations of the T-mesh, for any choice of the underlying extended complete Tchebycheff space.
\end{abstract}

Keywords: Extended complete Tchebycheff spaces, Tchebycheffian splines, T-meshes, dimension formula, dimension bounds, dimension instabilities

\section{Introduction}

Tensor-product structures allow us to construct multivariate splines in a very simple and elegant way from univariate splines, and they have been applied in different contexts. However, such a multivariate structure lacks adequate local refinement, which is imperative for both geometric modeling and numerical simulation. This triggered the interest in alternative multivariate spline structures supporting local refinement but still preserving locally the simplicity of the tensor-product approach. T-splines $[11,19]$, hierarchical splines $[7,8]$ and locally refined (LR-) splines [6] are examples of such structures. All of them can be seen as special instances of splines over T-meshes [5, 18].

Univariate Tchebycheffian splines are smooth piecewise functions with sections in extended Tchebycheff spaces $[15,17]$. They share many important properties with the classical (algebraic) polynomial splines but also offer a more flexible framework, due to the wide variety of extended Tchebycheff spaces. Within the large class of extended Tchebycheff spaces, extended complete Tchebycheff spaces are a particularly interesting subclass because they have some additional useful properties, such as the possibility of defining so-called generalized power functions [12]. As a consequence, beyond their theoretical interest, spline spaces with sections in extended complete Tchebycheff spaces are attractive in several application areas ranging from geometric modeling to isogeometric analysis (see, e.g., $[14,15]$ ). Multivariate extensions of Tchebycheffian splines can be easily obtained via (local) tensor-product structures.

Tchebycheffian spline spaces over T-meshes have been introduced in their full generality in [3]. Some earlier generalizations of the polynomial setting towards the Tchebycheffian setting were considered in $[2,4,13]$. Like in the polynomial case, a complete understanding of such Tchebycheffian spline spaces requires the knowledge of the dimension of the spline space defined on a prescribed T-mesh for a given smoothness. Unfortunately, in a complete analogy with the polynomial case, it turns out that the dimension of the spline space can be unstable. This means that the dimension may depend not only on combinatorial quantities of the T-mesh (such as number of vertices, edges and faces), on the given smoothness and on the componentwise dimensions of the underlying section spaces, but also on the exact geometry of the T-mesh. Such instabilities complicate the derivation of an explicit dimension formula for any T-mesh configuration, and only lower and upper bounds can be given in the most general cases.

Email addresses: cesare.bracco@unifi.it (Cesare Bracco), tom@math.uio.no (Tom Lyche), manni@mat. uniroma2. it (Carla Manni), speleers@mat. uniroma2.it (Hendrik Speleers) 
In [3] lower bounds for the dimension of Tchebycheffian spline spaces over T-meshes are provided by generalizing the homological techniques and the results presented in [16] for polynomials. Explicit upper bounds for the dimension are also obtained in [3] under a specific assumption on the underlying extended Tchebycheff spaces: the so-called $\boldsymbol{d}$-sum property. It has been proved in [16] that polynomial spaces possess this property but it is not known which other extended Tchebycheff spaces enjoy the $\boldsymbol{d}$-sum property as well.

In this paper we show that any extended complete Tchebycheff space possesses the $\boldsymbol{d}$-sum property, as conjectured in [3], and we link it to the minimal support property of univariate Tchebycheffian B-splines. This result allows us to apply the dimension upper bounds obtained in [16] to any extended complete Tchebycheff spline space over a planar T-mesh. The relevance of this result is threefold. First, the class of extended complete Tchebycheff spaces contains the most important Tchebycheff spaces from the application point of view, such as trigonometric and hyperbolic spaces. Second, the known upper and lower bounds agree on several relevant T-mesh configurations, resulting in explicit expressions for the dimension of the corresponding Tchebycheffian spline spaces. Third, this paves the path for a full generalization to the Tchebycheffian setting of the construction of LR-splines by providing a proper tool to address their linear independence.

Furthermore, we analyze instability in the dimension of extended complete Tchebycheffian spline spaces over T-meshes. Deepening the analysis presented in [3], we show that there exist T-meshes such that the corresponding spline spaces have unstable dimension for any choice of the underlying extended complete Tchebycheff space.

The remainder of the paper is organized as follows. In Section 2 we recall the definition and main properties of extended complete Tchebycheff spaces, and in Section 3 we prove that they possess the $\boldsymbol{d}$-sum property. Section 4 is devoted to the definition of Tchebycheffian spline spaces over T-meshes. In Section 5 we collect the main results on the dimension upper bounds and discuss some of their consequences. Examples of instability in the dimension of $C^{1}$ spline spaces over T-meshes for any underlying extended complete Tchebycheff space of dimension 3 are provided in Section 6 . We end with some concluding remarks in Section 7.

The derivative operator plays a crucial role throughout the paper. It will be denoted by $D$ whenever it is clear on which variable it operates. If this is not the case, we will use the notation $D_{x}, D_{y}$ to avoid any confusion.

\section{Extended complete Tchebycheff spaces}

We first define extended Tchebycheff spaces on a real interval $J$ (see, e.g., [17]).

Definition 2.1 (Extended Tchebycheff space). Given an integer $p \geq 0$ and an interval J, a space $\mathbb{T}_{p}(J) \subset C^{p}(J)$ of dimension $p+1$ is an extended Tchebycheff (ET-) space on $J$ if any Hermite interpolation problem with $p+1$ data on $J$ has a unique solution in $\mathbb{T}_{p}(J)$. In other words, for any integer $m \geq 1$, let $\bar{x}_{1}, \ldots, \bar{x}_{m}$ be distinct points in $J$ and let $d_{1}, \ldots, d_{m}$ be nonnegative integers such that $p+1=\sum_{i=1}^{m}\left(d_{i}+1\right)$. Then, for any set $\left\{f_{i, j} \in \mathbb{R}\right\}_{i=1, \ldots, m, j=0, \ldots, d_{i}}$ there exists a unique $q \in \mathbb{T}_{p}(J)$ such that

$$
D^{j} q\left(\bar{x}_{i}\right)=f_{i, j}, \quad i=1, \ldots, m, \quad j=0, \ldots, d_{i} .
$$

Example 2.1. The space $\mathbb{P}_{p}:=\left\langle 1, x, \ldots, x^{p}\right\rangle$ of algebraic polynomials of degree less than or equal to $p$ is an ET-space on the real line.

Example 2.2. The space $\langle\cos x, \sin x\rangle$ is an ET-space on any interval $[a, a+\pi)$.

We now consider a particular subclass of ET-spaces, whose properties will be crucial later on.

Definition 2.2 (Extended complete Tchebycheff system). Let $J$ be an interval of the real line. A set $\left\{u_{0}, \ldots, u_{p}\right\}$ of functions in $C^{p}(J)$ is an extended complete Tchebycheff (ECT-) system on $J$ if for all $x_{0} \leq x_{1} \leq \cdots \leq x_{k} \in J$ and $k=0, \ldots, p$ :

$$
\operatorname{det}\left(\begin{array}{llll}
x_{0} & x_{1} & \cdots & x_{k} \\
u_{0} & u_{1} & \cdots & u_{k}
\end{array}\right)>0
$$

where

$$
\left(\begin{array}{llll}
x_{0} & x_{1} & \cdots & x_{k} \\
u_{0} & u_{1} & \cdots & u_{k}
\end{array}\right)
$$

is the (Hermite) collocation matrix of the functions $u_{0}, \ldots, u_{k}$ at the points $x_{0}, \ldots, x_{k}$. 
By [17, Theorem 9.1] the set $\left\{u_{0}, \ldots, u_{p}\right\}$ of functions in $C^{p}(J)$ is an ECT-system if and only if their Wronskian determinants

$$
W\left(u_{0}, \ldots, u_{k}\right)(x):=\operatorname{det}\left(D^{i} u_{j}(x)\right)_{i, j=0}^{k}, \quad k=0, \ldots, p,
$$

are positive for all $x \in J$.

Definition 2.3 (Extended complete Tchebycheff space). An extended complete Tchebycheff (ECT-) space of dimension $p+1$ on $J$ is a space spanned by an ECT-system $\left\{u_{0}, \ldots, u_{p}\right\}$ on $J$.

From (2.1) with $k=p$ we have that an ECT-space of dimension $p+1$ on $J$ is an ET-space of dimension $p+1$ on $J$.

Example 2.3. The space $\mathbb{P}_{p}$ of algebraic polynomials of degree less than or equal to $p$ is an ECT-space on any interval of the real line. It can be seen as the span of the ECT-system

$$
\left\{1, x-c, \frac{(x-c)^{2}}{2}, \ldots, \frac{(x-c)^{p}}{p !}\right\}
$$

for any fixed $c \in \mathbb{R}$. Indeed, the Wronskian determinants of this system are all equal to one. The functions in (2.2) form the classical Taylor basis for algebraic polynomials.

Example 2.4. The space $\langle\cos x, \sin x\rangle$ is an ECT-space on the interval $(0, \pi)$ but not on $[0, \pi)$, where it is an ET-space, see Example 2.2.

According to [12], suppose $J$ is a finite interval and $w_{1}, \ldots, w_{p}$ are given continuous functions on $J$. For $x, y \in J$ we define repeated integrals by

$$
I_{k}\left(x, y ; w_{1}, \ldots, w_{k}\right):=\int_{y}^{x} w_{1}(t) I_{k-1}\left(t, y ; w_{2}, \ldots, w_{k}\right) \mathrm{d} t, \quad k \geq 1
$$

starting with $I_{0}(x, y):=1$ and $I_{-1}(x, y):=0$. We summarize some useful properties of these repeated integrals in the following lemma, see [12, Proposition 3.1].

Lemma 2.1. Suppose $p \geq 2, w_{k} \in C^{p-1}(J), k=1, \ldots, p$ and $x, y \in J$. Then,

$$
\begin{aligned}
& D_{x} I_{k}\left(x, y ; w_{1}, \ldots, w_{k}\right)=w_{1}(x) I_{k-1}\left(x, y ; w_{2}, \ldots, w_{k}\right) \\
& D_{y} I_{k}\left(x, y ; w_{1}, \ldots, w_{k}\right)=-w_{k}(y) I_{k-1}\left(x, y ; w_{1}, \ldots, w_{k-1}\right), \\
& \left.D_{x}^{r} I_{k}(x, y)\right|_{x=y}=0, \quad r=0, \ldots, k-1 \\
& I_{p}\left(x, y ; w_{1}, \ldots, w_{p}\right)=\sum_{k=0}^{p}(-1)^{p-k} I_{k}\left(x, c ; w_{1}, \ldots, w_{k}\right) I_{p-k}\left(y, c ; w_{p}, \ldots, w_{k+1}\right), \quad c \in J .
\end{aligned}
$$

Example 2.5. If $w_{i}=1$ for $i=1, \ldots, k$, then

$$
I_{k}\left(x, y ; w_{1}, \ldots, w_{k}\right)=\frac{(x-y)^{k}}{k !}
$$

In this case (2.7) takes the form

$$
\frac{(x-y)^{p}}{p !}=\sum_{k=0}^{p}(-1)^{p-k} \frac{(x-c)^{k}}{k !} \frac{(y-c)^{p-k}}{(p-k) !} .
$$

This is the binomial expansion of $(x-y)^{p} / p !=((x-c)-(y-c))^{p} / p !$.

With a proper choice of the functions $w_{1}, \ldots, w_{p}$, the above repeated integrals provide a characterization of any ECT-space. We first define the so-called generalized power functions

$$
u_{k}(x, c):=w_{0}(x) I_{k}\left(x, c ; w_{1}, \ldots, w_{k}\right), \quad k=0,1, \ldots, p .
$$

The following result can be found in [17, Chapter 9]. 
Theorem 2.1. The space $\mathbb{T}_{p}(J)$ is an ECT-space of dimension $p+1$ on $J$ if and only if there are positive functions $w_{k} \in C^{p}(J), k=0, \ldots, p$ such that for any $c \in J$ the generalized power functions (2.8) $\operatorname{span} \mathbb{T}_{p}(J)$.

Many properties of algebraic polynomials involve working with derivatives. When dealing with an ECT-system $\left\{u_{0}, \ldots, u_{p}\right\}$ as in $(2.8)$ it is convenient to replace the usual derivatives by some related differential operators $L_{k}, k=0, \ldots, p$ as follows. Let $w_{k} \in C^{p}(J), k=0, \ldots, p$ be positive functions on $J$. We define for sufficiently smooth functions $f$,

$$
L_{0} f:=f \text {, }
$$

and for $j \geq 1$,

$$
L_{j} f:=D\left(\frac{L_{j-1} f}{w_{j-1}}\right) .
$$

The operator $L_{j}$ can be regarded as a substitute of the classical derivative operator $D^{j}$. Indeed, a direct computation gives

$$
L_{j} f=\frac{D^{j} f}{w_{0} \cdots w_{j-1}}+\sum_{k=0}^{j-1} a_{j, k} D^{k} f
$$

for some functions $a_{j, 0}, \ldots, a_{j, j-1}$. Moreover, the following properties can be easily verified.

Lemma 2.2. We have

$$
\begin{gathered}
L_{j} u_{k}(x, c)=w_{j}(x) I_{k-j}\left(x, c ; w_{j+1}, \ldots, w_{k}\right), \quad j=0, \ldots, k, \\
L_{j} u_{k}(c, c)=0, \quad j<k, \\
D^{j} u_{k}(c, c)=0, \quad j<k .
\end{gathered}
$$

From Theorem 2.1 it follows that the $\operatorname{span} \mathbb{T}_{p}^{*}(J)$ of the functions

$$
u_{k}^{*}(x, c):=I_{k}\left(x, c ; w_{p}, \ldots, w_{p-k+1}\right), \quad k=0,1, \ldots, p
$$

is also an ECT-space of dimension $p+1$. This is called the dual space of $\mathbb{T}_{p}(J)$. For this space it is natural to define the differential operators $L_{0}^{*} f:=f$ and for $j \geq 1$,

$$
L_{j}^{*}:=\frac{1}{w_{p-j+1}} D L_{j-1}^{*} .
$$

When multiplying by $w_{0}(x)$, the relation $(2.7)$ can be written as

$$
u_{p}(x, y)=\sum_{k=0}^{p}(-1)^{p-k} u_{k}(x, c) I_{p-k}\left(y, c ; w_{p}, \ldots, w_{k+1}\right)=\sum_{k=0}^{p}(-1)^{p-k} u_{k}(x, c) u_{p-k}^{*}(y, c) .
$$

Assuming $L_{j}^{*}$ operates on the $y$ variable and by (2.4) we have

$$
\begin{aligned}
L_{j}^{*} u_{p}(x, y) & =\sum_{k=0}^{p}(-1)^{p-k} u_{k}(x, c) L_{j}^{*} I_{p-k}\left(y, c ; w_{p}, \ldots, w_{k+1}\right) \\
& =\sum_{k=0}^{p-j}(-1)^{p-k} u_{k}(x, c) I_{p-k-j}\left(y, c ; w_{p-j}, \ldots, w_{k+1}\right) .
\end{aligned}
$$

When taking $c=y$, we obtain

$$
L_{j}^{*} u_{p}(x, y)=(-1)^{j} u_{p-j}(x, y) .
$$

Example 2.6. For the algebraic polynomial space $\mathbb{P}_{p}$ we have $\mathbb{P}_{p}^{*}=\mathbb{P}_{p}$. In this case, with $w_{0}=\cdots=$ $w_{p}=1$, we have $L_{j}^{*}=L_{j}=D^{j}$ and

$$
u_{k}^{*}(x, c)=I_{k}\left(x, c ; w_{p}, \ldots, w_{p-k+1}\right)=\frac{(x-c)^{k}}{k !}=u_{k}(x, c), \quad k=0,1, \ldots, p .
$$

The equality (2.10) is the well-known binomial expansion of $(x-y)^{p} / p$ !, see Example 2.5. The equality (2.11) takes the form

$$
D_{y}^{j} \frac{(x-y)^{p}}{p !}=(-1)^{j} \frac{(x-y)^{p-j}}{(p-j) !} .
$$


Example 2.7. The space $\langle 1, \cos x, \sin x\rangle$ is an ECT-space on the interval $J=(-\pi, \pi)$, with

$$
w_{0}(x)=\cos ^{2}\left(\frac{x}{2}\right), \quad w_{1}(x)=w_{2}(x)=1 / \cos ^{2}\left(\frac{x}{2}\right) .
$$

Indeed, for $x \in J$ and any fixed $y \in J$ we find the following ECT-system spanning $\langle 1, \cos x, \sin x\rangle$ :

$$
\begin{aligned}
& u_{0}(x, y)=w_{0}(x) I_{0}(x, y)=\cos ^{2}\left(\frac{x}{2}\right) \\
& u_{1}(x, y)=w_{0}(x) I_{1}\left(x, y ; w_{1}\right)=\sin x-(1+\cos x) \tan \left(\frac{y}{2}\right) \\
& u_{2}(x, y)=w_{0}(x) I_{2}\left(x, y ; w_{1}, w_{2}\right)=2 \sin ^{2}\left(\frac{x-y}{2}\right) / \cos ^{2}\left(\frac{y}{2}\right),
\end{aligned}
$$

and the dual space is spanned by

$$
\begin{aligned}
& u_{0}^{*}(x, y)=I_{0}(x, y)=1 \\
& u_{1}^{*}(x, y)=I_{1}\left(x, y ; w_{2}\right)=2\left(\tan \left(\frac{x}{2}\right)-\tan \left(\frac{y}{2}\right)\right), \\
& u_{2}^{*}(x, y)=I_{2}\left(x, y ; w_{2}, w_{1}\right)=2\left(\tan \left(\frac{x}{2}\right)-\tan \left(\frac{y}{2}\right)\right)^{2} .
\end{aligned}
$$

These functions span the null space of the differential operators

$$
\begin{aligned}
& L_{3}=\cos ^{2}\left(\frac{x}{2}\right)\left(D^{3}+D\right), \\
& L_{3}^{*}=\frac{1}{2}\left((1+\cos x) D^{3}-3 \sin x D^{2}+(1-2 \cos x) D\right) .
\end{aligned}
$$

Moreover, for any $c \in(-\pi, \pi)$, (2.10) takes the form

$$
u_{2}(x, y)=u_{2}(x, c) u_{0}^{*}(y, c)-u_{1}(x, c) u_{1}^{*}(y, c)+u_{0}(x, c) u_{2}^{*}(y, c) .
$$

\section{3. $d$-sum property}

Consider an ET-space $\mathbb{T}_{p}(J)$ of dimension $p+1$ on $J$. Given $d \in \mathbb{N}$ and $\bar{x} \in J$, we define

$$
\mathbb{I}^{\mathbb{T}_{p}, d}(\bar{x}):=\left\{q \in \mathbb{T}_{p}(J): D^{l} q(\bar{x})=0, l=0, \ldots, d\right\}
$$

The following property is an important ingredient for the dimension results for Tchebycheffian spline spaces over T-meshes [3].

Definition 3.1 (d-sum property). Consider an ET-space $\mathbb{T}_{p}(J)$ of dimension $p+1$ on $J$. Let $\boldsymbol{d}:=$ $\left(d_{1}, \ldots, d_{m}\right)$ with $0 \leq d_{i} \leq p, d_{i} \in \mathbb{N}, i=1, \ldots, m$. We say that $\mathbb{T}_{p}(J)$ has the $\boldsymbol{d}$-sum property if for any set of $m$ distinct points $\bar{x}_{1}, \ldots, \bar{x}_{m} \in J$ we have

$$
\operatorname{dim}\left(\sum_{i=1}^{m} \mathbb{I}^{\mathbb{T}_{p}, d_{i}}\left(\bar{x}_{i}\right)\right)=\min \left(p+1, \sum_{i=1}^{m} p-d_{i}\right)
$$

where $\mathbb{I}^{\mathbb{T}_{p}, d_{i}}\left(\bar{x}_{i}\right)$ is defined in (3.1).

We start by showing that the space of algebraic polynomials has the $\boldsymbol{d}$-sum property. This has already been proved in [16, Proposition 1.8], but here we present an alternative proof where we provide some basic arguments which can be extended to prove the same property for ECT-spaces.

Lemma 3.1. Let $c_{0}, \ldots, c_{p}$ be distinct points in $\mathbb{R}$. Then, the functions

$$
\left(x-c_{0}\right)^{p}, \ldots,\left(x-c_{p}\right)^{p}
$$

are linearly independent and form a basis for $\mathbb{P}_{p}$. 
Proof. Let $c \in \mathbb{R}$ be fixed. Then,

$$
\frac{\left(x-c_{i}\right)^{p}}{p !}=\frac{\left(x-c+c-c_{i}\right)^{p}}{p !}=\sum_{k=0}^{p} \frac{(x-c)^{k}}{k !} \frac{\left(c_{i}-c\right)^{p-k}}{(p-k) !}(-1)^{p-k}
$$

The conversion matrix between the set $\left\{\left(x-c_{0}\right)^{p}, \ldots,\left(x-c_{p}\right)^{p}\right\}$ and the Taylor basis (see $\left.(2.2)\right)$ is non-singular because, up to a multiplication of every column by a proper factor, it is the collocation matrix of the Taylor basis at distinct points $c_{0}, \ldots, c_{p}$. Since $(2.2)$ is a basis for $\mathbb{P}_{p}$, it follows that also $\left\{\left(x-c_{0}\right)^{p}, \ldots,\left(x-c_{p}\right)^{p}\right\}$ is a basis for $\mathbb{P}_{p}$.

Theorem 3.1. The space of algebraic polynomials $\mathbb{P}_{p}$ has the $\boldsymbol{d}$-sum property for any $\boldsymbol{d}:=\left(d_{1}, \ldots, d_{m}\right)$ with $0 \leq d_{i} \leq p, d_{i} \in \mathbb{N}, i=1, \ldots, m$ and for any $m \in \mathbb{N}$.

Proof. If $d_{i}=p$ then $\mathbb{I}^{\mathbb{P}_{p}, d_{i}}\left(\bar{x}_{i}\right)=\{0\}$. Thus, it suffices to assume that $d_{i}<p$ without loss of generality. We define the following polynomials belonging to $\mathbb{P}_{p}$ :

$$
\eta_{i, j}(x):=D^{j} \frac{\left(x-\bar{x}_{i}\right)^{p}}{p !}=\frac{\left(x-\bar{x}_{i}\right)^{p-j}}{(p-j) !}, \quad i=1, \ldots, m, \quad j=0, \ldots, p-1-d_{i} .
$$

It is clear that, for each $i=1, \ldots, m$, the $p-d_{i}$ functions $\eta_{i, j}$ belong to $\mathbb{I}^{\mathbb{P}}, d_{i}\left(\bar{x}_{i}\right)$ since each of them has a factor $\left(x-\bar{x}_{i}\right)^{d_{i}+1}$ for $j=0, \ldots, p-1-d_{i}$. Moreover, it easy to see that these $p-d_{i}$ functions are linearly independent and span $\mathbb{I}^{\mathbb{P}}, d_{i}\left(\bar{x}_{i}\right)$. Therefore,

$$
\operatorname{dim}\left(\sum_{i=1}^{m} \mathbb{I}^{\mathbb{P}_{p}, d_{i}}\left(\bar{x}_{i}\right)\right) \leq \min \left(p+1, \sum_{i=1}^{m} p-d_{i}\right)
$$

In the remainder of the proof, we show the linear independence of (possibly a subset of) the functions in $(3.2)$.

First assume that $\sum_{i=1}^{m}\left(p-d_{i}\right)=p+1$. Given the polynomials

$$
\phi_{i, j} \in \mathbb{P}_{p}, \quad i=1, \ldots, m, \quad j=0, \ldots, p-1-d_{i},
$$

such that

$$
D^{l} \phi_{i, j}\left(\bar{x}_{k}\right)=\delta_{i, k} \delta_{j, l}, \quad k=1, \ldots, m, \quad l=0, \ldots, p-1-d_{k},
$$

we define

$$
u(x, y):=\sum_{i=1}^{m} \sum_{j=0}^{p-1-d_{i}}(-1)^{j} \phi_{i, j}(y) \eta_{i, j}(x) .
$$

Note that for $k=1, \ldots, m$ and $l=0, \ldots, p-1-d_{k}$,

$$
D_{y}^{l} u\left(x, \bar{x}_{k}\right)=\sum_{i=1}^{m} \sum_{j=0}^{p-1-d_{i}}(-1)^{j} D^{l} \phi_{i, j}\left(\bar{x}_{k}\right) \eta_{i, j}(x)=(-1)^{l} \eta_{k, l}(x)=\left.D_{y}^{l} \frac{(x-y)^{p}}{p !}\right|_{y=\bar{x}_{k}} .
$$

These are $\sum_{k=1}^{m}\left(p-d_{k}\right)=p+1$ Hermite conditions. From the uniqueness of Hermite interpolation in the $y$ variable we deduce that

$$
\frac{(x-y)^{p}}{p !}=u(x, y)=\sum_{i=1}^{m} \sum_{j=0}^{p-1-d_{i}}(-1)^{j} \phi_{i, j}(y) D^{j} \frac{\left(x-\bar{x}_{i}\right)^{p}}{p !} .
$$

This relation can be used to represent the set of basis functions of $\mathbb{P}_{p}$ described in Lemma 3.1. Since (3.4) holds for all $x, y$, it follows that the sets of $p+1$ polynomials in (3.2) and (3.3) both form a basis for $\mathbb{P}_{p}$. This implies that the $p+1$ functions in (3.2) are linearly independent and form a basis for $\sum_{i=1}^{m} \mathbb{I}_{p}^{\mathbb{P}_{p}, d_{i}}\left(\bar{x}_{i}\right)$

If $\sum_{i=1}^{m}\left(p-d_{i}\right)>p+1$ we consider integers $\bar{d}_{1}, \ldots, \bar{d}_{m}$ with $\bar{d}_{i} \geq d_{i}$ such that $\sum_{i=1}^{m}\left(p-\bar{d}_{i}\right)=p+1$. Thus, $\mathbb{I}^{\mathbb{P}_{p}, \bar{d}_{i}}\left(\bar{x}_{i}\right) \subset \mathbb{I}^{\mathbb{P}}, d_{i}\left(\bar{x}_{i}\right)$, and from the previous arguments it follows

$$
p+1 \geq \operatorname{dim}\left(\sum_{i=1}^{m} \mathbb{I}^{\mathbb{P}_{p}, d_{i}}\left(\bar{x}_{i}\right)\right) \geq \operatorname{dim}\left(\sum_{i=1}^{m} \mathbb{I}^{\mathbb{P}_{p}, \bar{d}_{i}}\left(\bar{x}_{i}\right)\right)=p+1 .
$$


If $\sum_{i=1}^{m}\left(p-d_{i}\right)<p+1$ we consider an additional point $\bar{x}_{m+1}$ and an integer $d_{m+1}$ so that $\sum_{i=1}^{m+1}(p-$ $\left.d_{i}\right)=p+1$. Using again the previous arguments it is clear that the functions in (3.2) are a subset of the linearly independent functions

$$
\left\{D^{j} \frac{\left(x-\bar{x}_{i}\right)^{p}}{p !}, \quad i=1, \ldots, m+1, \quad j=0, \ldots, p-1-d_{i}\right\} .
$$

Therefore, the $\sum_{i=1}^{m}\left(p-d_{i}\right)$ functions in (3.2) are linearly independent and form a basis for $\sum_{i=1}^{m} \mathbb{I}^{\mathbb{P}_{p}, d_{i}}\left(\bar{x}_{i}\right)$.

In the following, we will show that the $\boldsymbol{d}$-sum property holds for a large subclass of ET-spaces, namely the class of ECT-spaces. The next lemma extends Lemma 3.1 to the case of ECT-spaces, see also Examples 2.3 and 2.6.

Lemma 3.2. Let $c_{0}, \ldots, c_{p}$ be distinct points in $J$. Then, the functions

$$
u_{p}\left(x, c_{0}\right), \ldots, u_{p}\left(x, c_{p}\right)
$$

are linearly independent and form a basis for $\mathbb{T}_{p}(J)$.

Proof. Let $c \in J$ be fixed. From (2.10) we have

$$
u_{p}\left(x, c_{i}\right)=\sum_{k=0}^{p} u_{k}(x, c) u_{p-k}^{*}\left(c_{i}, c\right)(-1)^{p-k} .
$$

Then, we can complete the proof by following the same line of arguments as in the proof of Lemma 3.1 using Theorem 2.1.

We are now ready to prove the main result of this section.

Theorem 3.2. An ECT-space has the $\boldsymbol{d}$-sum property for any $\boldsymbol{d}:=\left(d_{1}, \ldots, d_{m}\right)$ with integers $d_{i}$ such that $0 \leq d_{i} \leq p$ for $i=1, \ldots, m$ and any $m \in \mathbb{N}$.

Proof. Suppose $\sum_{i=1}^{m}\left(p-d_{i}\right)=p+1$ with $d_{i}<p$. Since $\mathbb{T}_{p}^{*}(J)$ is an ET-space, it follows from (2.9) that for any given sufficiently smooth function $f$ on $J$ there is a unique function $g \in \mathbb{T}_{p}^{*}(J)$ such that

$$
L_{l}^{*} g\left(\bar{x}_{k}\right)=L_{l}^{*} f\left(\bar{x}_{k}\right), \quad k=1, \ldots, m, \quad l=0,1, \ldots p-1-d_{k} .
$$

This function $g$ can be written in the form

$$
g(y)=\sum_{i=1}^{m} \sum_{j=0}^{p-1-d_{i}} \phi_{i, j}^{*}(y) L_{j}^{*} f\left(\bar{x}_{i}\right)
$$

where $L_{j}^{*}$ operates on the $y$ variable and

$$
\phi_{i, j}^{*} \in \mathbb{T}_{p}^{*}, \quad i=1, \ldots, m, \quad j=0, \ldots, p-1-d_{i},
$$

are such that

$$
L_{l}^{*} \phi_{i, j}^{*}\left(\bar{x}_{k}\right)=\delta_{i, k} \delta_{j, l}, \quad k=1, \ldots, m, \quad l=0, \ldots, p-1-d_{k} .
$$

The functions $\phi_{i, j}^{*}$ exist as they are unique solutions of Hermite interpolation problems. Taking into account (2.11), it follows that for any fixed $x \in J$,

$$
u_{p}(x, y)=\sum_{i=1}^{m} \sum_{j=0}^{p-1-d_{i}} \phi_{i, j}^{*}(y) L_{j}^{*} u_{p}\left(x, \bar{x}_{i}\right)=\sum_{i=1}^{m} \sum_{j=0}^{p-1-d_{i}} \phi_{i, j}^{*}(y)(-1)^{j} u_{p-j}\left(x, \bar{x}_{i}\right) .
$$

Note that

$$
u_{p-j}\left(x, \bar{x}_{i}\right) \in \mathbb{I}^{\mathbb{T}_{p}, d_{i}}\left(\bar{x}_{i}\right), \quad j=0, \ldots, p-1-d_{i} .
$$

Indeed, for $l=0, \ldots, d_{i}$ and $j=0, \ldots, p-1-d_{i}$ we have $p-j \geq d_{i}+1>l$, and from Lemma 2.2 we know

$$
D^{l} u_{p-j}\left(\bar{x}_{i}, \bar{x}_{i}\right)=0 .
$$

Moreover, from Lemma 3.2 we know that the functions $u_{p}\left(x, c_{0}\right), \ldots, u_{p}\left(x, c_{p}\right)$ are linearly independent for distinct points $c_{0}, \ldots, c_{p} \in J$. Then, we can complete the proof by following the same line of arguments as in the proof of Theorem 3.1 . 
We now show that the $\boldsymbol{d}$-sum property is related to an important property of Tchebycheffian splines. Let $J:=[a, b]$, the partition

$$
\Delta:=\left\{a=\bar{x}_{0}<\bar{x}_{1}<\cdots<\bar{x}_{l+1}=b\right\}
$$

and the vector of integers $\boldsymbol{d}:=\left(d_{1}, \ldots, d_{l}\right)$ with $-1 \leq d_{i}<p$ for $i=1, \ldots, l$ be given. We define the intervals $J_{i}:=\left[\bar{x}_{i}, \bar{x}_{i+1}\right), i=0, \ldots, l-1, J_{l}:=\left[\bar{x}_{l}, \bar{x}_{l+1}\right]$, and consider the space of Tchebycheffian splines on $\Delta$ :

$$
\mathbb{S}^{\mathbb{T}_{p}, \boldsymbol{d}}(\Delta):=\left\{s:[a, b] \rightarrow \mathbb{R}: s \in \mathbb{T}_{p}\left(J_{i}\right), i=0, \ldots, l, s \in C^{d_{i}}\left(\bar{x}_{i}\right), i=1, \ldots, l\right\} .
$$

From (2.6) and Theorem 2.1 it can be deduced that any element $s \in \mathbb{S}^{\mathbb{T}_{p}, \boldsymbol{d}}(\Delta)$ can be written in the form

$$
s(x)=\sum_{j=0}^{p} \alpha_{0, j} u_{j}\left(x, \bar{x}_{0}\right)+\sum_{i=1}^{l} \sum_{j=d_{i}+1}^{p} \alpha_{i, j} u_{j}\left(x, \bar{x}_{i}\right)_{+}, \quad \alpha_{i, j} \in \mathbb{R},
$$

where $u_{j}\left(x, \bar{x}_{i}\right)$ are the generalized power functions in (2.8), and for $x, \bar{x}_{i} \in[a, b]$,

$$
u_{j}\left(x, \bar{x}_{i}\right)_{+}:=\left\{\begin{array}{ll}
u_{j}\left(x, \bar{x}_{i}\right), & \text { if } x \geq \bar{x}_{i} \\
0, & \text { if } x<\bar{x}_{i}
\end{array},\right.
$$

are the so-called generalized truncated power functions. Note that $(2.6) \operatorname{implies} u_{j}\left(\cdot, \bar{x}_{i}\right)_{+} \in C^{j-1}$.

Example 3.1. The space $\mathbb{S}^{\mathbb{P}_{p}, \boldsymbol{d}}(\Delta)$ is the classical space of algebraic polynomial splines. In this case, the functions $u_{j}\left(x, \bar{x}_{i}\right)$ are $\left(x-\bar{x}_{i}\right)^{j} / j$ !, see Example 2.6, and the formula in (3.5) takes the form of the usual polynomial truncated power representation.

The next proposition links the $\boldsymbol{d}$-sum property to the minimal support of a nontrivial element in the space of Tchebycheffian splines.

Proposition 3.1. Let $s$ be an element in $\mathbb{S}^{\mathbb{T}}, \boldsymbol{d}(\Delta)$ such that

$$
s(x)=0, \quad x \notin\left[\bar{x}_{k}, \bar{x}_{k+m}\right],
$$

for some integers $k, m$ with $1 \leq k \leq l-m$. If $\sum_{i=k}^{k+m}\left(p-d_{i}\right) \leq p+1$ then $s$ is identically zero on $[a, b]$.

Proof. We proceed by contradiction. Suppose that $\sum_{i=k}^{k+m}\left(p-d_{i}\right) \leq p+1$ and $s$ is not identically zero on $[a, b]$. From $(3.5)$ we obtain

$$
s(x)=\sum_{i=k}^{k+m} \sum_{j=d_{i}+1}^{p} \alpha_{i, j} u_{j}\left(x, \bar{x}_{i}\right)_{+},
$$

for some coefficients $\alpha_{i, j}$ not all zero. Let $x \geq \bar{x}_{k+m}$. Then, we have $u_{j}\left(x, \bar{x}_{i}\right)_{+}=u_{j}\left(x, \bar{x}_{i}\right)$ for $i=k, \ldots, k+m$. Moreover, the functions $u_{j}\left(x, \bar{x}_{i}\right), j=d_{i}+1, \ldots, p$ span $\mathbb{T}^{\mathbb{T}}, d_{i}\left(\bar{x}_{i}\right)$, and so the set of functions

$$
\left\{u_{j}\left(x, \bar{x}_{i}\right), i=k, \ldots, k+m, j=d_{i}+1, \ldots, p\right\}
$$

$\operatorname{span} \sum_{i=k}^{k+m} \mathbb{I}^{\mathbb{T}_{p}, d_{i}}\left(\bar{x}_{i}\right)$. Since $\sum_{i=k}^{k+m}\left(p-d_{i}\right) \leq p+1$, Theorem 3.2 implies that

$$
\operatorname{dim}\left(\sum_{i=k}^{k+m} \mathbb{I}^{\mathbb{T}_{p}, d_{i}}\left(\bar{x}_{i}\right)\right)=\sum_{i=k}^{k+m} p-d_{i}
$$

For $x>\bar{x}_{k+m}$ we have $s(x)=0$, and so $\sum_{i=k}^{k+m} \sum_{j=d_{i}+1}^{p} \alpha_{i, j} u_{j}\left(x, \bar{x}_{i}\right)=0$. Hence, the elements of (3.6) are linearly dependent on $\left(\bar{x}_{k+m}, b\right)$, and as a consequence also on $[a, b]$ since they belong to an ET-space. This contradicts (3.7) and establishes the proposition.

Remark 3.1. In (3.5) we have seen that the generalized truncated power functions form a possible basis for $\mathbb{S}^{\mathbb{T}}, \boldsymbol{d}(\Delta)$. A more popular basis is given by the so-called Tchebycheffian B-splines, see [17, Chapter 9]. They enjoy several nice properties. In particular, they have minimal support, see [17, Lemma 9.21]. Proposition 3.1 shows that the $\boldsymbol{d}$-sum property is equivalent to the minimal support property of Tchebycheffian B-splines. Hence, our proof of the $\boldsymbol{d}$-sum property provides an alternative proof of the minimal support property of Tchebycheffian B-splines. Conversely, the d-sum property can also be deduced from [17, Lemma 9.21]. 


\section{Tchebycheffian spline spaces over T-meshes}

We first recall the concepts and definitions related to T-meshes, using the notation given in [3] (see also $[2,16])$. We consider a domain $\Omega \subset \mathbb{R}^{2}$ which is a finite union of closed axis-aligned rectangles, called cells, whose interiors are pairwise disjoint. This domain $\Omega$ is assumed to be simply connected and its interior $\Omega^{o}$ is connected. We denote by $\left[a_{h}, b_{h}\right] \times\left[a_{v}, b_{v}\right]$ the smallest rectangle containing $\Omega$.

Definition 4.1 (T-mesh). $A$ T-mesh $\mathcal{T}:=\left(\mathcal{T}_{2}, \mathcal{T}_{1}, \mathcal{T}_{0}\right)$ on $\Omega$ is defined as:

- $\mathcal{T}_{2}$ is the collection of cells in $\Omega$;

- $\mathcal{T}_{1}=\mathcal{T}_{1}^{h} \cup \mathcal{T}_{1}^{v}$ is a finite set of closed axis-aligned horizontal and vertical segments in $\bigcup_{\sigma \in \mathcal{T}_{2}} \partial \sigma$, called edges;

- $\mathcal{T}_{0}:=\bigcup_{\tau \in \mathcal{T}_{1}} \partial \tau$ is a finite set of points, called vertices;

such that

- for each $\sigma \in \mathcal{T}_{2}, \partial \sigma$ is a finite union of elements of $\mathcal{T}_{1}$;

- for $\sigma, \sigma^{\prime} \in \mathcal{T}_{2}$ with $\sigma \neq \sigma^{\prime}, \sigma \cap \sigma^{\prime}=\partial \sigma \cap \partial \sigma^{\prime}$ is a finite union of elements of $\mathcal{T}_{1} \cup \mathcal{T}_{0}$;

- for $\tau, \tau^{\prime} \in \mathcal{T}_{1}$ with $\tau \neq \tau^{\prime}, \tau \cap \tau^{\prime}=\partial \tau \cap \partial \tau^{\prime} \subset \mathcal{T}_{0}$;

- for each $\gamma \in \mathcal{T}_{0}, \gamma=\tau_{h} \cap \tau_{v}$ where $\tau_{h}$ is a horizontal edge and $\tau_{v}$ is a vertical edge.

We denote by $\mathcal{T}_{1}^{o}$ the set of interior edges, i.e., the edges intersecting $\Omega^{o}$. Analogously, $\mathcal{T}_{0}^{o}$ represents the set of interior vertices, i.e., the vertices in $\Omega^{o}$. The elements of the sets $\mathcal{T}_{1} \backslash \mathcal{T}_{1}^{o}$ and $\mathcal{T}_{0} \backslash \mathcal{T}_{0}^{o}$ are the boundary edges and the boundary vertices, respectively. Moreover, $\mathcal{T}_{1}^{o, h}$ and $\mathcal{T}_{1}^{o, v}$ indicate the sets of the horizontal and vertical interior edges of $\mathcal{T}$, respectively, and we set $\mathcal{T}_{1}^{o}:=\mathcal{T}_{1}^{o, h} \cup \mathcal{T}_{1}^{o, v}$. Then, the interior T-mesh is $\mathcal{T}^{o}:=\left(\mathcal{T}_{2}, \mathcal{T}_{1}^{o}, \mathcal{T}_{0}^{o}\right)$.

A segment of $\mathcal{T}$ is a connected union of edges of $\mathcal{T}$ belonging to the same straight line. Given any $\tau \in \mathcal{T}_{1}^{o}$, we denote by $\rho(\tau)$ the maximal segment composed of edges of $\mathcal{T}_{1}^{o}$ containing $\tau$. Moreover, we denote by $\operatorname{MS}(\mathcal{T})$ the set of all such maximal segments, and by $\operatorname{MIS}(\mathcal{T})$ the set of all maximal interior segments $(M I S)$, that is the subset of $\operatorname{MS}(\mathcal{T})$ whose elements do not intersect the boundary of the T-mesh. The set of all horizontal (respectively vertical) maximal interior segments is denoted by $\operatorname{MIS}_{h}(\mathcal{T})$ (respectively $\operatorname{MIS}_{v}(\mathcal{T})$ ). Given any $\gamma \in \mathcal{T}_{0}^{o}$, we define $\rho_{h}(\gamma):=\rho\left(\tau_{h}\right)$ and $\rho_{v}(\gamma):=\rho\left(\tau_{v}\right)$, such that $\gamma=\tau_{h} \cap \tau_{v}$ and $\tau_{h} \in \mathcal{T}_{1}^{o, h}, \tau_{v} \in \mathcal{T}_{1}^{o, v}$.

Since we are going to deal with Tchebycheffian spline spaces over T-meshes, we also need to formalize the concept of smoothness in this context.

Definition 4.2 (Smoothness). With each edge $\tau \in \mathcal{T}_{1}^{o}$, we associate an integer $r(\tau) \geq 0$. We say that $f \in C^{r(\tau)}(\tau)$ if the partial derivatives of $f$ up to order $r(\tau)$ are continuous across the edge $\tau$. We assume that $r(\tau)=r\left(\tau^{\prime}\right)$ for all $\tau, \tau^{\prime}$ lying on the same straight line, and we refer to this as the constant smoothness (along lines) assumption. A smoothness distribution on $\mathcal{T}$ is defined as

$$
\boldsymbol{r}:=\left\{r(\tau), \forall \tau \in \mathcal{T}_{1}^{o}\right\},
$$

and leads to the following class of smooth functions on $\Omega$ :

$$
C^{\boldsymbol{r}}(\mathcal{T}):=\left\{f: \Omega \rightarrow \mathbb{R}: f \in C^{r(\tau)}(\tau), \forall \tau \in \mathcal{T}_{1}^{o}\right\} .
$$

Given a smoothness distribution $\boldsymbol{r}$ on $\mathcal{T}$, with each vertex $\gamma \in \mathcal{T}_{0}^{o}$, we associate two integers $r_{h}(\gamma), r_{v}(\gamma)$, where $r_{h}(\gamma):=r\left(\tau_{v}\right)$ and $r_{v}(\gamma):=r\left(\tau_{h}\right)$ such that $\gamma=\tau_{h} \cap \tau_{v}$ and $\tau_{h} \in \mathcal{T}_{1}^{o, h}, \tau_{v} \in \mathcal{T}_{1}^{o, v}$. For each maximal segment $\rho \in \operatorname{MS}(\mathcal{T})$ we set $r(\rho):=r(\tau)$, where $\tau$ is any interior edge belonging to $\rho$.

In the following, we denote by $\ell$ either $h$ or $v$. Let $p_{\ell} \in \mathbb{N}$ with $p_{\ell} \geq 0$, and let $\mathbb{T}_{p_{\ell}}^{\ell}\left(\left[a_{\ell}, b_{\ell}\right]\right)$ be an ET-space of dimension $p_{\ell}+1$ on $J_{\ell}:=\left[a_{\ell}, b_{\ell}\right]$. Then, we define the tensor-product space

$$
\mathbb{P}_{\boldsymbol{p}}^{\boldsymbol{T}}:=\mathbb{T}_{p_{h}}^{h}\left(\left[a_{h}, b_{h}\right]\right) \otimes \mathbb{T}_{p_{v}}^{v}\left(\left[a_{v}, b_{v}\right]\right),
$$

where $\boldsymbol{p}:=\left(p_{h}, p_{v}\right)$ and $\boldsymbol{T}:=\left(T_{h}, T_{v}\right):=\left(\mathbb{T}_{p_{h}}^{h}, \mathbb{T}_{p_{v}}^{v}\right)$. If the space (4.1) is the space of bivariate algebraic polynomials of bi-degree $\boldsymbol{p}$, then it is denoted by $\mathbb{P}_{\boldsymbol{p}}$. In analogy with the polynomial case, we refer to $\boldsymbol{p}$ as the bi-degree of the space $\mathbb{P}_{\boldsymbol{p}}^{T}$. 
Definition 4.3 (Tchebycheffian spline space over a T-mesh). Let $\mathcal{T}$ be a T-mesh with a smoothness distribution $\boldsymbol{r}$, and let $p_{h}, p_{v} \in \mathbb{N}$ with $p_{h}, p_{v} \geq 0$. The (extended) Tchebycheffian spline space over the T-mesh $\mathcal{T}$, denoted by $\mathbb{S}_{\boldsymbol{p}}^{\boldsymbol{T}, \boldsymbol{r}}(\mathcal{T})$, is defined as the space of functions in $C^{\boldsymbol{r}}(\mathcal{T})$ such that, restricted to any cell $\sigma \in \mathcal{T}_{2}$, they belong to $\mathbb{P}_{\boldsymbol{p}}^{\boldsymbol{T}}$, i.e.,

$$
\mathbb{S}_{\boldsymbol{p}}^{\boldsymbol{T}, \boldsymbol{r}}(\mathcal{T}):=\left\{s \in C^{\boldsymbol{r}}(\mathcal{T}): s_{\mid \sigma} \in \mathbb{P}_{\boldsymbol{p}}^{\boldsymbol{T}}, \sigma \in \mathcal{T}_{2}\right\}
$$

The space $\mathbb{S}_{\boldsymbol{p}}^{\boldsymbol{T}, \boldsymbol{r}}(\mathcal{T})$ is called extended complete Tchebycheffian spline space over $\mathcal{T}$ if $\mathbb{P}_{\boldsymbol{p}}^{\boldsymbol{T}}$ is a tensorproduct of two ECT-spaces. It is called polynomial spline space over $\mathcal{T}$ if $\mathbb{P}_{\boldsymbol{p}}^{\boldsymbol{T}}=\mathbb{P}_{\boldsymbol{p}}$ and denoted by $\mathbb{S}_{\boldsymbol{p}}^{\boldsymbol{r}}(\mathcal{T})$.

In the following, we will assume the usual condition on the smoothness (see [3, Section 2.2])

$$
r\left(\tau_{v}\right)<p_{h}, \quad \forall \tau_{v} \in \mathcal{T}_{1}^{o, v}, \quad r\left(\tau_{h}\right)<p_{v}, \quad \forall \tau_{h} \in \mathcal{T}_{1}^{o, h}
$$

For some detailed examples of T-meshes and the related concepts, we refer to [3, Section 2] and [2, Section 2].

\section{Dimension formula for extended complete Tchebycheffian spline spaces over T-meshes}

In [3] homological techniques were employed to obtain bounds for the dimension of extended Tchebycheffian spline spaces $\mathbb{S}_{\boldsymbol{p}}^{\boldsymbol{T}, \boldsymbol{r}}(\mathcal{T})$ defined over a T-mesh $\mathcal{T}$. In this section, we improve those results for extended complete Tchebycheffian spline spaces (see Theorem 5.1). Thanks to Theorem 3.2, we are able to formulate the bounds, without any assumption on the relation between the bi-degree of the space and its smoothness. Let us first recall some preliminary definitions and concepts.

Definition 5.1 ( $\boldsymbol{r}$-sum property on $\mathcal{T})$. Given a smoothness distribution $\boldsymbol{r}$ on $\mathcal{T}$, we say that $\boldsymbol{T}:=$ $\left(\mathbb{T}_{p_{h}}^{h}, \mathbb{T}_{p_{v}}^{v}\right)$ has the $\boldsymbol{r}$-sum property on $\mathcal{T}$, if each of its components $\mathbb{T}_{p_{\ell}}^{\ell}\left(\left[a_{\ell}, b_{\ell}\right]\right)$ with $\ell=h$, $v$ has the $\boldsymbol{d}$-sum property (see Definition 3.1) for any subvector $\boldsymbol{d}$ of the vector $\boldsymbol{r}_{\ell}:=\left(r_{\ell}(\gamma)\right)_{\gamma \in \mathcal{T}_{0}^{o}}$.

Let $\iota$ be an ordering of $\operatorname{MIS}(\mathcal{T})$. For any $\rho \in \operatorname{MIS}(\mathcal{T})$, we denote by $\Gamma_{\iota}(\rho)$ the set of vertices of $\rho$ which do not belong to $\rho^{\prime} \in \operatorname{MIS}(\mathcal{T})$ with $\iota\left(\rho^{\prime}\right)>\iota(\rho)$.

Definition 5.2 (Weight of MIS). Given an ordering $\iota$ of $\operatorname{MIS}(\mathcal{T})$, the weight of a maximal interior segment $\rho \in \operatorname{Mis}(\mathcal{T})$ is defined as

$$
\omega_{\iota}(\rho):=\left\{\begin{array}{ll}
\sum_{\gamma \in \Gamma_{\iota}(\rho)}\left(p_{h}-r_{h}(\gamma)\right), & \text { if } \rho \in \operatorname{MIS}_{h}(\mathcal{T}) \\
\sum_{\gamma \in \Gamma_{\iota}(\rho)}\left(p_{v}-r_{v}(\gamma)\right), & \text { if } \rho \in \operatorname{MIS}_{v}(\mathcal{T})
\end{array} .\right.
$$

In the next theorem we collect the dimension results for extended complete Tchebycheffian spline spaces over T-meshes. They are a full generalization of the results known for the polynomial case [16, Theorems 3.1 and 3.7].

Theorem 5.1. Let $\mathbb{S}_{\boldsymbol{p}}^{\boldsymbol{T}, \boldsymbol{r}}(\mathcal{T})$ be an extended complete Tchebycheffian spline space over a $T$-mesh $\mathcal{T}$. Then,

$$
\begin{aligned}
\operatorname{dim}\left(\mathbb{S}_{\boldsymbol{p}}^{\boldsymbol{T}, \boldsymbol{r}}(\mathcal{T})\right) & =\sum_{\sigma \in \mathcal{T}_{2}}\left(p_{h}+1\right)\left(p_{v}+1\right)-\sum_{\tau \in \mathcal{T}_{1}^{o, h}}\left(p_{h}+1\right)(r(\tau)+1)-\sum_{\tau \in \mathcal{T}_{1}^{o, v}}(r(\tau)+1)\left(p_{v}+1\right) \\
& +\sum_{\gamma \in \mathcal{T}_{0}^{o}}\left(r_{h}(\gamma)+1\right)\left(r_{v}(\gamma)+1\right)+H_{0}
\end{aligned}
$$

where

$$
0 \leq H_{0} \leq \sum_{\rho \in \operatorname{MIS}_{h}(\mathcal{T})}\left(p_{h}+1-\omega_{\iota}(\rho)\right)_{+}\left(p_{v}-r(\rho)\right)+\sum_{\rho \in \operatorname{MIS}_{v}(\mathcal{T})}\left(p_{h}-r(\rho)\right)\left(p_{v}+1-\omega_{\iota}(\rho)\right)_{+},
$$

and $(x)_{+}:=\max (x, 0)$. 


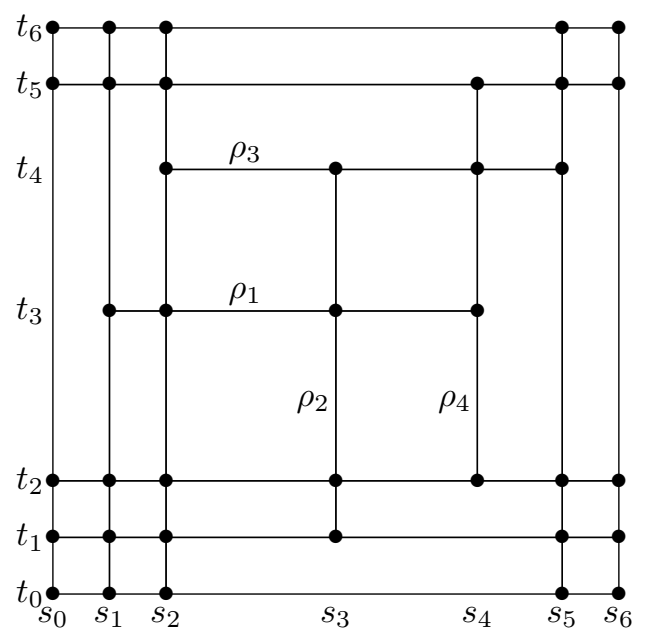

Figure 1: Example of an unstable T-mesh.

Proof. The dimension formula (5.1) was shown in [3, Theorem 4.1] with $H_{0}$ a specific homology term. Furthermore, in [3, Theorem 3.1], it was proved that such homology term can be bounded by (5.2) under the assumption that the considered couple of ET-spaces $\boldsymbol{T}=\left(\mathbb{T}_{p_{h}}^{h}, \mathbb{T}_{p_{v}}^{v}\right)$ satisfies the $\boldsymbol{r}$-sum property on $\mathcal{T}$. Since $\mathbb{S}_{\boldsymbol{p}}^{\boldsymbol{T}, \boldsymbol{r}}(\mathcal{T})$ is an extended complete Tchebycheffian spline space, we know from Theorem 3.2 that this is the case, and the proof is complete.

Example 5.1. Consider the T-mesh $\mathcal{T}$ in Figure 1. This mesh has 4 maximal interior segments, namely $\operatorname{MIS}(\mathcal{T})=\operatorname{MIS}_{h}(\mathcal{T}) \cup \operatorname{MIS}_{v}(\mathcal{T})$ with $\operatorname{MIS}_{h}(\mathcal{T})=\left\{\rho_{1}, \rho_{3}\right\}$ and $\operatorname{MIS}_{v}(\mathcal{T})=\left\{\rho_{2}, \rho_{4}\right\}$. The ordering $\iota$ of $\operatorname{MIS}(\mathcal{T})$ is given by $\iota\left(\rho_{j}\right)=j, j=1,2,3,4$. Note that

$$
\left|\mathcal{T}_{2}\right|=24, \quad\left|\mathcal{T}_{1}^{o, h}\right|=\left|\mathcal{T}_{1}^{o, v}\right|=22, \quad\left|\mathcal{T}_{0}^{o}\right|=21
$$

Moreover, let $\boldsymbol{p}=\left(p_{h}, p_{v}\right)=(2,2)$ and let $\boldsymbol{r}$ be the constant smoothness distribution where $r(\tau)=1$ for all $\tau \in \mathcal{T}_{1}^{o}$. Then,

$$
\omega_{\iota}\left(\rho_{1}\right)=2, \quad \omega_{\iota}\left(\rho_{2}\right)=3, \quad \omega_{\iota}\left(\rho_{3}\right)=3, \quad \omega_{\iota}\left(\rho_{4}\right)=4 .
$$

Theorem 5.1 implies that $0 \leq H_{0} \leq 1$, and $36 \leq \operatorname{dim}\left(\mathbb{S}_{\boldsymbol{p}}^{\boldsymbol{T}, \boldsymbol{r}}(\mathcal{T})\right) \leq 37$ for $\boldsymbol{T}=\left(\mathbb{T}_{2}^{h}, \mathbb{T}_{2}^{v}\right)$ where $\mathbb{T}_{2}^{h}, \mathbb{T}_{2}^{v}$ are any ECT-spaces of dimension 3 on the intervals $\left[s_{0}, s_{6}\right]$ and $\left[t_{0}, t_{6}\right]$, respectively.

Theorem 5.1 gives an explicit and computable expression for the dimension of the space $\mathbb{S}_{\boldsymbol{p}}^{\boldsymbol{T}, \boldsymbol{r}}(\mathcal{T})$ when the upper bound in (5.2) is zero (and so $H_{0}=0$ ). It is evident that such configurations are of practical interest, and therefore the upper bound in (5.2) plays an important role in the design of T-mesh refinement algorithms. This upper bound depends on the bi-degree, on the smoothness, and on the weights of MIS with respect to any ordering of MIS. If the T-mesh is obtained by successive refinements, we can use the following algorithm (Algorithm 5.1) to generate a naturally induced ordering of MIS, which will be employed to construct a T-mesh refinement algorithm (Algorithm 5.2) ensuring $H_{0}=0$.

Algorithm 5.1. Given a T-mesh $\mathcal{T}$ with an ordering $\iota$ of $\operatorname{MIS}(\mathcal{T})$, let $\tilde{\mathcal{T}}$ be the $T$-mesh obtained after inserting a new segment $\tau$ (i.e., one or more consecutive edges). The ordering $\tilde{\iota}$ of $\operatorname{MIS}(\tilde{\mathcal{T}})$ is computed as follows:

1. set $\tilde{\iota}(\tilde{\rho}):=\iota(\tilde{\rho})$ for all $\tilde{\rho} \in \operatorname{MIS}(\tilde{\mathcal{T}}) \backslash\{\rho(\tau)\}$ where $\rho(\tau) \in \operatorname{MS}(\tilde{\mathcal{T}})$ is the maximal segment containing $\tau$;

2. if $\rho(\tau) \in \operatorname{Mis}(\tilde{\mathcal{T}})$ :

(a) if $\rho(\tau)=\rho^{\prime} \cup \tau \cup \rho^{\prime \prime}$ with $\rho^{\prime}, \rho^{\prime \prime} \in \operatorname{MIS}(\mathcal{T})$, then $\tilde{\iota}(\rho(\tau)):=\min \left(\iota\left(\rho^{\prime}\right), \iota\left(\rho^{\prime \prime}\right)\right)$;

(b) if $\rho(\tau)=\tau \cup \rho^{\prime}$ with $\rho^{\prime} \in \operatorname{Mis}(\mathcal{T})$, then $\tilde{\iota}(\rho(\tau)):=\iota\left(\rho^{\prime}\right)$;

(c) if $\rho(\tau)=\tau$, then $\tilde{\iota}(\tau):=1+\max _{\rho^{\prime} \in \operatorname{MIS}(\mathcal{T})} \iota\left(\rho^{\prime}\right)$. 


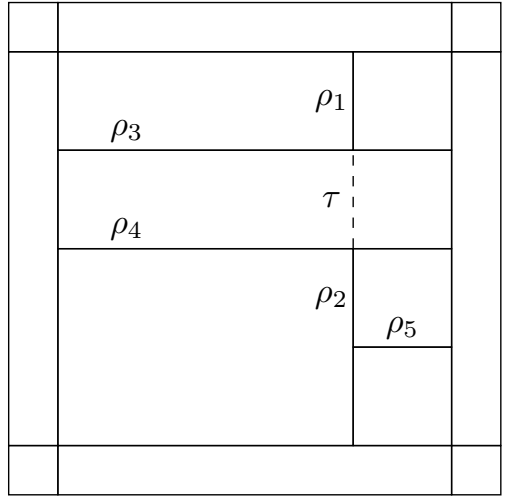

(a) $\tau$ links two existing MIS $\left(\rho(\tau)=\rho_{1} \cup \tau \cup \rho_{2}\right)$

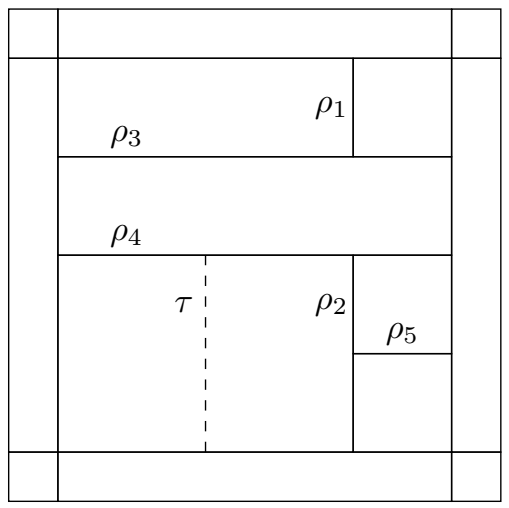

(c) $\tau$ introduces a new MIS $(\rho(\tau)=\tau)$

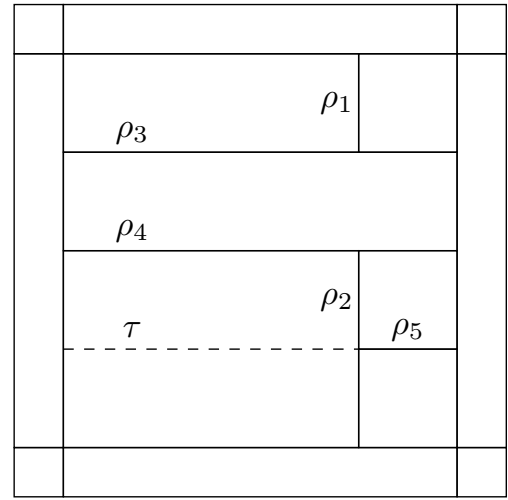

(b) $\tau$ extends an existing MIS $\left(\rho(\tau)=\tau \cup \rho_{5}\right)$

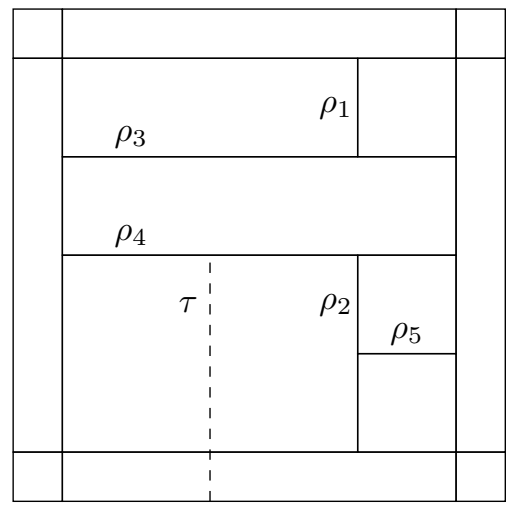

(d) $\tau$ does not modify the set of MIS $(\rho(\tau)=\tau)$

Figure 2: Examples of the different cases of inserting a segment $\tau$ (indicated by dashed lines) in Algorithm 5.1.

Figure 2 illustrates the different cases of inserting a new segment $\tau$ in Algorithm 5.1. Note that the indices of the ordering of MIS produced by Algorithm 5.1 are not necessarily consecutive. As shown in the next lemma, Algorithm 5.1 ensures that the weights of the already existing MIS do not decrease.

Lemma 5.1. Given a T-mesh $\mathcal{T}$ with an ordering $\iota$ of $\operatorname{MIS}(\mathcal{T})$, let $\tilde{\mathcal{T}}$ be the $T$-mesh obtained after inserting a new segment $\tau$ and let $\tilde{\imath}$ be the ordering of $\operatorname{MIS}(\tilde{\mathcal{T}})$ computed by Algorithm 5.1. Then, for all $\tilde{\rho} \in \operatorname{MiS}(\tilde{\mathcal{T}})$ such that $M(\tilde{\rho}):=\{\rho \in \operatorname{MIS}(\mathcal{T}): \rho \subseteq \tilde{\rho}\} \neq \emptyset$, there exists $\bar{\rho} \in M(\tilde{\rho})$ with $\iota(\bar{\rho})=\tilde{\iota}(\tilde{\rho})$ and $\omega_{\iota}(\bar{\rho}) \leq \omega_{\tilde{\iota}}(\tilde{\rho})$.

Proof. Let us first focus on Step 1 in $\operatorname{Algorithm} 5$.1. Since $\operatorname{MIS}(\tilde{\mathcal{T}}) \backslash\{\rho(\tau)\}=\operatorname{MIS}(\tilde{\mathcal{T}}) \cap \operatorname{MIS}(\mathcal{T})$, we have $M(\tilde{\rho})=\{\tilde{\rho}\}$ for all $\tilde{\rho} \in \operatorname{Mis}(\tilde{\mathcal{T}}) \backslash\{\rho(\tau)\}$. In such case, we set $\bar{\rho}=\tilde{\rho}$. When arriving at Step $2($ a) and considering $\tilde{\rho}=\rho(\tau) \in \operatorname{MIS}(\tilde{\mathcal{T}})$, we have $M(\tilde{\rho})=\left\{\rho^{\prime}, \rho^{\prime \prime}\right\}$ and we set $\bar{\rho}=\rho^{\prime}$ if $\iota\left(\rho^{\prime}\right)<\iota\left(\rho^{\prime \prime}\right)$ and $\bar{\rho}=\rho^{\prime \prime}$ otherwise. Similarly, at Step 2(b), we have $M(\tilde{\rho})=\left\{\rho^{\prime}\right\}$ and we set $\bar{\rho}=\rho^{\prime}$. Note that we can ignore Step 2(c) since $M(\tilde{\rho})=\emptyset$.

For the above choices of $\bar{\rho}$ it is clear from the algorithm that $\iota(\bar{\rho})=\tilde{\iota}(\tilde{\rho})$. In the last part of the proof, we will show that $\Gamma_{\iota}(\bar{\rho}) \subseteq \Gamma_{\tilde{\iota}}(\tilde{\rho})$, and this immediately implies $\omega_{\iota}(\bar{\rho}) \leq \omega_{\tilde{\iota}}(\tilde{\rho})$. Inserting $\tau$ results in adding possible vertices belonging to the elements of $\operatorname{MIS}(\mathcal{T})$. Therefore, by the definition of $\Gamma_{\iota}, \Gamma_{\tilde{\iota}}$ and $\iota(\bar{\rho})=\tilde{\iota}(\tilde{\rho})$, we have $\Gamma_{\iota}(\bar{\rho})=\Gamma_{\tilde{\iota}}(\tilde{\rho})$ if $\tilde{\iota}(\tilde{\rho})<\tilde{\iota}(\rho(\tau))$, and $\Gamma_{\iota}(\bar{\rho}) \subseteq \Gamma_{\tilde{\iota}}(\tilde{\rho})$ otherwise, see also Figure 2.

We now detail a refinement strategy that generates a sequence of refined T-meshes such that the upper bound in (5.2) is kept to be zero throughout the entire refinement process. It is a particular implementation of the so-called $\left(p_{h}+1, p_{v}+1\right)$-weighted subdivision rule described in [16, Algorithm 4.4]. 
This rule was developed in the context of polynomial splines but is also valid in our more general Tchebycheffian spline setting.

Algorithm 5.2. Given a T-mesh $\mathcal{T}$ with an ordering $\iota$ of $\operatorname{MIS}(\mathcal{T})$, two positive integers $p_{h}, p_{v}$, and a smoothness distribution $\boldsymbol{r}$ such that $\omega_{\iota}(\rho) \geq p_{h}+1$ for any $\rho \in \operatorname{MIS}_{h}(\mathcal{T})$ and $\omega_{\iota}(\rho) \geq p_{v}+1$ for any $\rho \in \operatorname{MIS}_{v}(\mathcal{T})$, we construct the refinement as follows for a segment $\tau$ to be inserted in $\mathcal{T}$ :

1. if $\tau$ does not extend an existing edge, then extend $\tau$ so that the maximal segment containing $\tau$, say $\rho(\tau)$, intersects $\partial \Omega$ or satisfies

$$
\sum_{\rho^{\prime} \in \Gamma_{\rho(\tau)}}\left(p_{\tau}-r\left(\rho^{\prime}\right)\right) \geq p_{\tau}+1, \quad p_{\tau}:= \begin{cases}p_{h}, & \text { if } \tau \text { is horizontal }, \\ p_{v}, & \text { if } \tau \text { is vertical }\end{cases}
$$

where $\Gamma_{\rho(\tau)}$ is the set of maximal segments $\rho^{\prime} \in \operatorname{MS}(\mathcal{T})$ intersecting $\rho(\tau)$;

2. update the ordering $\iota$ according to Algorithm 5.1 .

The next proposition shows that Algorithm 5.2 can be applied successively once a valid initial T-mesh configuration is constructed. For example, any tensor-product mesh leads to a valid initial configuration because it does not contain MIS. Moreover, Algorithm 5.2 guarantees that $H_{0}=0$ in Theorem 5.1.

Proposition 5.1. Let $\tilde{\mathcal{T}}$ be the T-mesh generated by Algorithm 5.2 and let $\tilde{\iota}$ be the corresponding ordering of $\operatorname{Mis}(\tilde{\mathcal{T}})$. We have

- $\omega_{\tilde{\iota}}(\tilde{\rho}) \geq p_{h}+1$ for any $\tilde{\rho} \in \operatorname{MIS}_{h}(\tilde{\mathcal{T}})$ and $\omega_{\tilde{\iota}}(\tilde{\rho}) \geq p_{v}+1$ for any $\tilde{\rho} \in \operatorname{MiS}_{v}(\tilde{\mathcal{T}}) ;$

- $H_{0}=0$.

Proof. Let $\tau$ be the segment to be inserted in $\mathcal{T}$ by means of Algorithm 5.2. If $\tau$ does not extend an existing edge and $\rho(\tau) \in \operatorname{Mis}(\tilde{\mathcal{T}})$, then Algorithm 5.1 (Step 2(c)) gives $\tilde{\iota}(\tau):=1+\max _{\rho^{\prime} \in \operatorname{mis}(\mathcal{T})} \iota\left(\rho^{\prime}\right)$. Hence, $\Gamma_{\tilde{\iota}}(\rho(\tau))$ is the set of interior vertices of $\tilde{\mathcal{T}}$ belonging to $\rho(\tau)$, i.e., the intersections of $\rho(\tau)$ with the elements in $\Gamma_{\rho(\tau)}$. By Definition 5.2 and by the condition (5.3), we get $\omega_{\tilde{\iota}}(\rho(\tau)) \geq p_{h}+1$ if $\rho(\tau) \in \operatorname{MIS}_{h}(\tilde{\mathcal{T}})$ and $\omega_{\tilde{\iota}}(\rho(\tau)) \geq p_{v}+1$ if $\rho(\tau) \in \operatorname{MIS}_{v}(\tilde{\mathcal{T}})$. Moreover, from the properties of Algorithm 5.1 in Lemma 5.1 we know that the weight of any other MIS in $\tilde{\mathcal{T}}$, say $\tilde{\rho}$, is not decreased (because $M(\tilde{\rho}) \neq \emptyset)$. It follows that $\omega_{\tilde{\iota}}(\tilde{\rho}) \geq p_{h}+1$ for any $\tilde{\rho} \in \operatorname{MIS}_{h}(\tilde{\mathcal{T}})$ and $\omega_{\tilde{\iota}}(\tilde{\rho}) \geq p_{v}+1$ for any $\tilde{\rho} \in \operatorname{MIS}_{v}(\tilde{\mathcal{T}})$. By using the bounds in (5.2), this also implies that $H_{0}=0$.

The refinement strategy detailed in Algorithm 5.2 and the corresponding explicit dimension formula is completely in agreement with similar results given for polynomial LR B-splines in [6, Section 5] and for polynomial spline spaces over T-meshes in [10, Section 4].

Theorem 5.1 also further clarifies the relationship between Tchebycheffian and polynomial spline spaces over T-meshes. When comparing the dimensions of an extended complete Tchebycheffian spline space $\mathbb{S}_{\boldsymbol{p}}^{\boldsymbol{T}, \boldsymbol{r}}(\mathcal{T})$ with the related polynomial spline space $\mathbb{S}_{\boldsymbol{p}}^{\boldsymbol{r}}(\mathcal{T})$, the difference between the two dimensions is bounded as

$$
\begin{aligned}
\left|\operatorname{dim}\left(\mathbb{S}_{\boldsymbol{p}}^{\boldsymbol{T}, \boldsymbol{r}}(\mathcal{T})\right)-\operatorname{dim}\left(\mathbb{S}_{\boldsymbol{p}}^{\boldsymbol{r}}(\mathcal{T})\right)\right| & \leq \sum_{\rho \in \mathrm{MIS}_{h}(\mathcal{T})}\left(p_{h}+1-\omega_{\iota}(\rho)\right)_{+}\left(p_{v}-r(\rho)\right) \\
& +\sum_{\rho \in \mathrm{MIS}_{v}(\mathcal{T})}\left(p_{h}-r(\rho)\right)\left(p_{v}+1-\omega_{\iota}(\rho)\right)_{+}
\end{aligned}
$$

In particular, such difference is zero if the mesh $\mathcal{T}$ has been obtained by applying Algorithm 5.2.

\section{Instability}

In this section we show that the dimension of the extended complete Tchebycheffian spline space $\mathbb{S}_{\boldsymbol{p}}^{\boldsymbol{T}, \boldsymbol{r}}(\mathcal{T})$ can depend not only on the topological information of $\mathcal{T}$ but also on the geometry of the $\mathrm{T}$ mesh. This particular behavior is usually referred to as instability in the dimension of the considered space. Examples of instability in the dimension of spline spaces over T-meshes are well known for polynomial spline spaces [1,9] and for trigonometric and hyperbolic spline spaces [3]. 
We focus on the T-mesh in Figure 1, which is a mirrored version of the one already considered in $[3,9]$. We consider $\boldsymbol{p}=(2,2)$ and a constant smoothness distribution $\boldsymbol{r}$ such that $r(\tau)=1$ for all $\tau \in \mathcal{T}_{1}^{o}$. Moreover, we set $\boldsymbol{T}=\left(\mathbb{T}_{2}, \mathbb{T}_{2}\right)$, where $\mathbb{T}_{2}$ is an ECT-space of dimension 3 on a suitable interval, identified by the positive weights $w_{0}, w_{1}, w_{2}$, see Theorem 2.1. From Example 5.1 we have

$$
\operatorname{dim}\left(\mathbb{S}_{\boldsymbol{p}}^{\boldsymbol{T}, \boldsymbol{r}}(\mathcal{T})\right)=36+H_{0}, \quad 0 \leq H_{0} \leq 1
$$

Following the same reasoning as in [3, Section 5], we also know that $H_{0}=12-K_{0}$, where $K_{0}$ is the dimension of the space spanned by the rows of the following matrix

$$
M:=\left(\begin{array}{cccc}
\psi_{s_{4}, 2}(x) & 0 & 0 & \psi_{t_{3}, 2}(y) \\
\psi_{s_{1}, 2}(x) & 0 & 0 & 0 \\
\psi_{s_{2}, 2}(x) & 0 & 0 & 0 \\
\psi_{s_{3}, 2}(x) & \psi_{t_{3}, 2}(y) & 0 & 0 \\
0 & \psi_{t_{1}, 2}(y) & 0 & 0 \\
0 & \psi_{t_{2}, 2}(y) & 0 & 0 \\
0 & \psi_{t_{4}, 2}(y) & \psi_{s_{3}, 2}(x) & 0 \\
0 & 0 & \psi_{s_{2}, 2}(x) & 0 \\
0 & 0 & \psi_{s_{5}, 2}(x) & 0 \\
0 & 0 & \psi_{s_{4}, 2}(x) & \psi_{t_{4}, 2}(y) \\
0 & 0 & 0 & \psi_{t_{2}, 2}(y) \\
0 & 0 & 0 & \psi_{t_{5}, 2}(y)
\end{array}\right) .
$$

In this matrix, $\left\{\psi_{\bar{z}, i}\right\}_{i=0}^{2}$ is a Taylor-like basis of the space $\mathbb{T}_{2}$, i.e.,

$$
D_{z}^{k} \psi_{\bar{z}, i}(\bar{z})=\delta_{i k}, \quad i, k=0,1,2 .
$$

For example, in the polynomial case (i.e., $\mathbb{T}_{2}=\mathbb{P}_{2}$ ) we have

$$
\psi_{\bar{z}, i}(z)=\frac{(z-\bar{z})^{i}}{i !}, \quad i=0,1,2
$$

and $K_{0}$ is given by the dimension of the space spanned by the rows of the following matrix

$$
M_{\mathbb{P}_{2}}\left(s_{1}, \ldots, s_{5} ; t_{1}, \ldots, t_{5}\right):=\left(\begin{array}{cccccccccccc}
1 & s_{4} & s_{4}{ }^{2} & 0 & 0 & 0 & 0 & 0 & 0 & 1 & t_{3} & t_{3}{ }^{2} \\
1 & s_{1} & s_{1}{ }^{2} & 0 & 0 & 0 & 0 & 0 & 0 & 0 & 0 & 0 \\
1 & s_{2} & s_{2}{ }^{2} & 0 & 0 & 0 & 0 & 0 & 0 & 0 & 0 & 0 \\
1 & s_{3} & s_{3}{ }^{2} & 1 & t_{3} & t_{3}{ }^{2} & 0 & 0 & 0 & 0 & 0 & 0 \\
0 & 0 & 0 & 1 & t_{1} & t_{1}{ }^{2} & 0 & 0 & 0 & 0 & 0 & 0 \\
0 & 0 & 0 & 1 & t_{2} & t_{2}{ }^{2} & 0 & 0 & 0 & 0 & 0 & 0 \\
0 & 0 & 0 & 1 & t_{4} & t_{4}{ }^{2} & 1 & s_{3} & s_{3}{ }^{2} & 0 & 0 & 0 \\
0 & 0 & 0 & 0 & 0 & 0 & 1 & s_{2} & s_{2}{ }^{2} & 0 & 0 & 0 \\
0 & 0 & 0 & 0 & 0 & 0 & 1 & s_{5} & s_{5}{ }^{2} & 0 & 0 & 0 \\
0 & 0 & 0 & 0 & 0 & 0 & 1 & s_{4} & s_{4}{ }^{2} & 1 & t_{4} & t_{4}{ }^{2} \\
0 & 0 & 0 & 0 & 0 & 0 & 0 & 0 & 0 & 1 & t_{2} & t_{2}{ }^{2} \\
0 & 0 & 0 & 0 & 0 & 0 & 0 & 0 & 0 & 1 & t_{5} & t_{5}{ }^{2}
\end{array}\right)
$$

It is clear that $\operatorname{rank}\left(M_{\mathbb{P}_{2}}\left(s_{1}, \ldots, s_{5} ; t_{1}, \ldots, t_{5}\right)\right) \geq 11$. The matrix $M_{\mathbb{P}_{2}}\left(s_{1}, \ldots, s_{5} ; t_{1}, \ldots, t_{5}\right)$ has been analyzed in [9] where it has been proved that it is singular if and only if

$$
\frac{\left(s_{3}-s_{1}\right)\left(s_{5}-s_{4}\right)}{\left(t_{3}-t_{1}\right)\left(t_{5}-t_{4}\right)}=\frac{\left(s_{4}-s_{1}\right)\left(s_{5}-s_{3}\right)}{\left(t_{4}-t_{1}\right)\left(t_{5}-t_{3}\right)}
$$

and in particular, if $s_{i}=t_{i}$ for all $i$. Hence, for $\boldsymbol{T}=\left(\mathbb{P}_{2}, \mathbb{P}_{2}\right)$ we get

$$
H_{0}= \begin{cases}1, & \text { if }(6.3) \text { holds, } \\ 0, & \text { otherwise }\end{cases}
$$

As a consequence, the dimension of the $C^{1}$ bi-quadratic polynomial spline space over the T-mesh in Figure 1 depends on the geometry of $\mathcal{T}$ according to the validity of (6.3). 
Let us now consider a general ECT-space $\mathbb{T}_{2}$. From Theorem 2.1 and from (2.6), (2.8) it follows that (possibly up to a constant) the Taylor-like basis function $\psi_{c, 2}$ is given by

$$
u_{2}(x, c)=w_{0}(x) I_{2}\left(x, c ; w_{1}, w_{2}\right) .
$$

Since $w_{0}>0$, the matrix in (6.2) has the same rank as the matrix

$$
M:=\left(\begin{array}{cccc}
I_{2}\left(x, s_{4} ; w_{1}, w_{2}\right) & 0 & 0 & I_{2}\left(y, t_{3} ; w_{1}, w_{2}\right) \\
I_{2}\left(x, s_{1} ; w_{1}, w_{2}\right) & 0 & 0 & 0 \\
I_{2}\left(x, s_{2} ; w_{1}, w_{2}\right) & 0 & 0 & 0 \\
I_{2}\left(x, s_{3} ; w_{1}, w_{2}\right) & I_{2}\left(y, t_{3} ; w_{1}, w_{2}\right) & 0 & 0 \\
0 & I_{2}\left(y, t_{1} ; w_{1}, w_{2}\right) & 0 & 0 \\
0 & I_{2}\left(y, t_{2} ; w_{1}, w_{2}\right) & 0 & 0 \\
0 & I_{2}\left(y, t_{4} ; w_{1}, w_{2}\right) & I_{2}\left(x, s_{3} ; w_{1}, w_{2}\right) & 0 \\
0 & 0 & I_{2}\left(x, s_{2} ; w_{1}, w_{2}\right) & 0 \\
0 & 0 & I_{2}\left(x, s_{5} ; w_{1}, w_{2}\right) & I_{2}\left(y, t_{4} ; w_{1}, w_{2}\right) \\
0 & 0 & I_{2}\left(x, s_{4} ; w_{1}, w_{2}\right) & I_{2} \\
0 & 0 & 0 & I_{2}\left(y, t_{2} ; w_{1}, w_{2}\right) \\
0 & 0 & 0 & I_{2}\left(y, t_{5} ; w_{1}, w_{2}\right)
\end{array}\right) .
$$

Using (2.7) we can write

$$
I_{2}\left(x, s_{i} ; w_{1}, w_{2}\right)=I_{2}\left(s_{i}, s_{1} ; w_{2}, w_{1}\right)-I_{1}\left(x, s_{1} ; w_{1}\right) I_{1}\left(s_{i}, s_{1} ; w_{2}\right)+I_{2}\left(x, s_{1} ; w_{1}, w_{2}\right), \quad i=1, \ldots, 5,
$$

and similarly

$$
I_{2}\left(y, t_{i} ; w_{1}, w_{2}\right)=I_{2}\left(t_{i}, t_{1} ; w_{2}, w_{1}\right)-I_{1}\left(y, t_{1} ; w_{1}\right) I_{1}\left(t_{i}, t_{1} ; w_{2}\right)+I_{2}\left(y, t_{1} ; w_{1}, w_{2}\right), \quad i=1, \ldots, 5 .
$$

Since $\left\{I_{k}\left(x, s_{1} ; w_{1}, \ldots, w_{k}\right), k=0,1,2\right\}$ (and also $\left.\left\{I_{k}\left(y, t_{1} ; w_{1}, \ldots, w_{k}\right), k=0,1,2\right\}\right)$ are linearly independent, $K_{0}$ is given by the dimension of the space spanned by the rows of the following matrix

$$
M_{\mathbb{T}_{2}}\left(s_{1}, \ldots, s_{5} ; t_{1}, \ldots, t_{5}\right):=\left(\begin{array}{cccccccccccc}
1 & S_{4}^{(1)} & S_{4}^{(2)} & 0 & 0 & 0 & 0 & 0 & 0 & 1 & T_{3}^{(1)} & T_{3}^{(2)} \\
1 & S_{1}^{(1)} & S_{1}^{(2)} & 0 & 0 & 0 & 0 & 0 & 0 & 0 & 0 & 0 \\
1 & S_{2}^{(1)} & S_{2}^{(2)} & 0 & 0 & 0 & 0 & 0 & 0 & 0 & 0 & 0 \\
1 & S_{3}^{(1)} & S_{3}^{(2)} & 1 & T_{3}^{(1)} & T_{3}^{(2)} & 0 & 0 & 0 & 0 & 0 & 0 \\
0 & 0 & 0 & 1 & T_{1}^{(1)} & T_{1}^{(2)} & 0 & 0 & 0 & 0 & 0 & 0 \\
0 & 0 & 0 & 1 & T_{2}^{(1)} & T_{2}^{(2)} & 0 & 0 & 0 & 0 & 0 & 0 \\
0 & 0 & 0 & 1 & T_{4}^{(1)} & T_{4}^{(2)} & 1 & S_{3}^{(1)} & S_{3}^{(2)} & 0 & 0 & 0 \\
0 & 0 & 0 & 0 & 0 & 0 & 1 & S_{2}^{(1)} & S_{2}^{(2)} & 0 & 0 & 0 \\
0 & 0 & 0 & 0 & 0 & 0 & 1 & S_{5}^{(1)} & S_{5}^{(2)} & 0 & 0 & 0 \\
0 & 0 & 0 & 0 & 0 & 0 & 1 & S_{4}^{(1)} & S_{4}^{(2)} & 1 & T_{4}^{(1)} & T_{4}^{(2)} \\
0 & 0 & 0 & 0 & 0 & 0 & 0 & 0 & 0 & 1 & T_{2}^{(1)} & T_{2}^{(2)} \\
0 & 0 & 0 & 0 & 0 & 0 & 0 & 0 & 0 & 1 & T_{5}^{(1)} & T_{5}^{(2)}
\end{array}\right) .
$$

where

$$
\begin{aligned}
& S_{i}^{(1)}:=I_{1}\left(s_{i}, s_{1} ; w_{2}\right)=\int_{s_{1}}^{s_{i}} w_{2}(v) \mathrm{d} v, \quad S_{i}^{(2)}:=I_{2}\left(s_{i}, s_{1} ; w_{2}, w_{1}\right)=\int_{s_{1}}^{s_{i}} w_{2}(v) \int_{s_{1}}^{v} w_{1}(u) \mathrm{d} u \mathrm{~d} v \\
& T_{i}^{(1)}:=I_{1}\left(t_{i}, t_{1} ; w_{2}\right)=\int_{t_{1}}^{t_{i}} w_{2}(v) \mathrm{d} v, \quad T_{i}^{(2)}:=I_{2}\left(t_{i}, t_{1} ; w_{2}, w_{1}\right) \int_{t_{1}}^{t_{i}} w_{2}(v) \int_{t_{1}}^{v} w_{1}(u) \mathrm{d} u \mathrm{~d} v
\end{aligned}
$$

In the case $w_{1}=w_{2}$, the rank of this matrix behaves exactly like in the polynomial case described by the following proposition.

Proposition 6.1. If $w_{1}=w_{2}$ then the matrix (6.4) is singular if and only if

$$
\frac{\left(S_{3}^{(1)}-S_{1}^{(1)}\right)\left(S_{5}^{(1)}-S_{4}^{(1)}\right)}{\left(T_{3}^{(1)}-T_{1}^{(1)}\right)\left(T_{5}^{(1)}-T_{4}^{(1)}\right)}=\frac{\left(S_{4}^{(1)}-S_{1}^{(1)}\right)\left(S_{5}^{(1)}-S_{3}^{(1)}\right)}{\left(T_{4}^{(1)}-T_{1}^{(1)}\right)\left(T_{5}^{(1)}-T_{3}^{(1)}\right)} .
$$


Proof. Let $w:=w_{1}=w_{2}$. By a symmetry argument we immediately have that

$$
S_{i}^{(2)}:=\int_{s_{1}}^{s_{i}} w(v) \int_{s_{1}}^{v} w(u) \mathrm{d} u \mathrm{~d} v=\frac{1}{2}\left(\int_{s_{1}}^{s_{i}} w(v) \mathrm{d} v\right)^{2}=\frac{1}{2}\left(S_{i}^{(1)}\right)^{2} .
$$

The result follows by comparing the matrices $M_{\mathbb{T}_{2}}\left(s_{1}, \ldots, s_{5} ; t_{1}, \ldots, t_{5}\right)$ and $M_{\mathbb{P}_{2}}\left(s_{1}, \ldots, s_{5} ; t_{1}, \ldots, t_{5}\right)$ and from (6.3).

Proposition 6.1 shows that, in the case $w_{1}=w_{2}$, the dimension of the Tchebycheffian spline space over the T-mesh in Figure 1 depends on the geometry of $\mathcal{T}$ according to the validity of (6.5).

We now deal with the general case of (possibly different) positive weights $w_{1}, w_{2}$.

Proposition 6.2. We have

$$
\operatorname{det}\left(M_{\mathbb{T}_{2}}\left(s_{1}, s_{2}, s_{3}, s_{4}, s_{5} ; s_{1}, s_{2}, s_{3}, s_{4}, s_{5}\right)\right)=0 .
$$

Moreover, for any pair $w_{1}, w_{2}$ there exists $\tilde{t}_{5}\left(w_{1}, w_{2}\right)>s_{5}$ such that for any $t_{5}$ with $s_{5}<t_{5}<\tilde{t}_{5}\left(w_{1}, w_{2}\right)$ we have

$$
\operatorname{det}\left(M_{\mathbb{T}_{2}}\left(s_{1}, s_{2}, s_{3}, s_{4}, s_{5} ; s_{1}, s_{2}, s_{3}, s_{4}, t_{5}\right)\right) \neq 0 \text {. }
$$

Proof. Since $s_{i}=t_{i}$ for $i=1,2,3,4$ we have

$$
T_{i}^{(1)}=S_{i}^{(1)}, \quad T_{i}^{(2)}=S_{i}^{(2)}, \quad i=1,2,3,4 .
$$

Assuming $t_{5}=s_{5}+\epsilon_{5}$ for some $\epsilon_{5} \geq 0$, we get

$$
T_{5}^{(1)}=S_{5}^{(1)}+\epsilon^{(1)}, \quad T_{5}^{(2)}=S_{5}^{(2)}+\epsilon^{(2)},
$$

where

$$
\epsilon^{(1)}=\int_{s_{5}}^{s_{5}+\epsilon_{5}} w_{2}(v) \mathrm{d} v, \quad \epsilon^{(2)}=\int_{s_{5}}^{s_{5}+\epsilon_{5}} w_{2}(v) \mathrm{d} v \int_{s_{1}}^{s_{5}} w_{1}(u) \mathrm{d} u+\int_{s_{5}}^{s_{5}+\epsilon_{5}} w_{2}(v) \int_{s_{5}}^{v} w_{1}(u) \mathrm{d} u \mathrm{~d} v .
$$

A direct computation gives

$$
\begin{aligned}
\operatorname{det}\left(M_{\mathbb{T}_{2}}\left(s_{1}, s_{2}, s_{3}, s_{4}, s_{5} ; s_{1}, s_{2}, s_{3}, s_{4}, t_{5}\right)\right)= & \\
& \left(S_{1}^{(1)} S_{3}^{(2)}-S_{2}^{(1)} S_{3}^{(2)}+S_{2}^{(1)} S_{1}^{(2)}-S_{1}^{(1)} S_{2}^{(2)}-S_{3}^{(1)} S_{1}^{(2)}+S_{3}^{(1)} S_{2}^{(2)}\right) \\
& \left(-S_{4}^{(1)} S_{1}^{(2)}+S_{4}^{(1)} S_{2}^{(2)}+S_{1}^{(1)} S_{4}^{(2)}-S_{2}^{(1)} S_{4}^{(2)}+S_{2}^{(1)} S_{1}^{(2)}-S_{1}^{(1)} S_{2}^{(2)}\right) \\
& \left(-S_{3}^{(1)} S_{4}^{(2)}+S_{2}^{(1)} S_{4}^{(2)}+S_{4}^{(1)} S_{3}^{(2)}-S_{2}^{(1)} S_{3}^{(2)}+S_{3}^{(1)} S_{2}^{(2)}-S_{4}^{(1)} S_{2}^{(2)}\right) \\
& \left(\epsilon^{(1)}\left(S_{5}^{(2)}-S_{2}^{(2)}\right)-\epsilon^{(2)}\left(S_{5}^{(1)}-S_{2}^{(1)}\right)\right),
\end{aligned}
$$

and so $\operatorname{det}\left(M_{\mathbb{T}_{2}}\left(s_{1}, s_{2}, s_{3}, s_{4}, s_{5} ; s_{1}, s_{2}, s_{3}, s_{4}, s_{5}\right)\right)=0$.

Suppose now $\epsilon_{5}>0$. We can rewrite the determinant as follows

$$
\begin{aligned}
\operatorname{det}\left(M_{\mathbb{T}_{2}}\left(s_{1}, s_{2}, s_{3}, s_{4}, s_{5} ; s_{1}, s_{2}, s_{3}, s_{4}, t_{5}\right)\right)=- & \left(S_{2}^{(1)}-S_{1}^{(1)}\right)\left(S_{3}^{(1)}-S_{1}^{(1)}\right)\left(R_{1,3}-R_{1,2}\right) \\
& \left(S_{2}^{(1)}-S_{1}^{(1)}\right)\left(S_{4}^{(1)}-S_{1}^{(1)}\right)\left(R_{1,4}-R_{1,2}\right) \\
& \left(S_{3}^{(1)}-S_{2}^{(1)}\right)\left(S_{4}^{(1)}-S_{2}^{(1)}\right)\left(R_{2,4}-R_{2,3}\right) \\
& \epsilon^{(1)}\left(S_{5}^{(1)}-S_{2}^{(1)}\right)\left(R_{2,5}-\epsilon^{(2)} / \epsilon^{(1)}\right),
\end{aligned}
$$

where

$$
R_{i, j}:=\frac{S_{j}^{(2)}-S_{i}^{(2)}}{S_{j}^{(1)}-S_{i}^{(1)}}, \quad i \neq j .
$$

Note that $R_{i, j}$ is well defined because $w_{2}(x)>0$, and

$$
\begin{aligned}
R_{i, j} & =\frac{\int_{s_{1}}^{s_{j}} w_{2}(v) \int_{s_{1}}^{v} w_{1}(u) \mathrm{d} u \mathrm{~d} v-\int_{s_{1}}^{s_{i}} w_{2}(v) \int_{s_{1}}^{v} w_{1}(u) \mathrm{d} u \mathrm{~d} v}{\int_{s_{i}}^{s_{j}} w_{2}(v) \mathrm{d} v} \\
& =\frac{\int_{s_{i}}^{s_{j}} w_{2}(v) \mathrm{d} v \int_{s_{1}}^{s_{i}} w_{1}(u) \mathrm{d} u+\int_{s_{i}}^{s_{j}} w_{2}(v) \int_{s_{i}}^{v} w_{1}(u) \mathrm{d} u \mathrm{~d} v}{\int_{s_{i}}^{s_{j}} w_{2}(v) \mathrm{d} v}=\int_{s_{1}}^{s_{i}} w_{1}(u) \mathrm{d} u+F\left(s_{i}, s_{j}\right),
\end{aligned}
$$


where

$$
F(x, y):=\frac{\int_{x}^{y} w_{2}(v) \int_{x}^{v} w_{1}(u) \mathrm{d} u \mathrm{~d} v}{\int_{x}^{y} w_{2}(v) \mathrm{d} v}
$$

Similarly, from (6.6) we obtain

$$
\frac{\epsilon^{(2)}}{\epsilon^{(1)}}=\int_{s_{1}}^{s_{5}} w_{1}(u) \mathrm{d} u+F\left(s_{5}, s_{5}+\epsilon_{5}\right) .
$$

It is clear that $S_{j}^{(1)}-S_{i}^{(1)}>0$ for $s_{i}<s_{j}$. Moreover, since $F(x, y)$ is increasing with respect to $y$ for any fixed $x, x<y$ (see Lemma 6.1), we deduce for $s_{i}<s_{j}<s_{k}$,

$$
R_{i, k}-R_{i, j}=F\left(s_{i}, s_{k}\right)-F\left(s_{i}, s_{j}\right)>0,
$$

and for sufficiently small but positive $\epsilon_{5}$, we get

$$
R_{2,5}-\frac{\epsilon^{(2)}}{\epsilon^{(1)}}=F\left(s_{2}, s_{5}\right)-\int_{s_{2}}^{s_{5}} w_{1}(u) \mathrm{d} u-F\left(s_{5}, s_{5}+\epsilon_{5}\right) \neq 0 .
$$

Summarizing, there exists $\tilde{t}_{5}\left(w_{1}, w_{2}\right)>s_{5}$ such that for any $t_{5}$ with $s_{5}<t_{5}<\tilde{t}_{5}\left(w_{1}, w_{2}\right)$ all the factors in $\operatorname{det}\left(M_{\mathbb{T}_{2}}\left(s_{1}, s_{2}, s_{3}, s_{4}, s_{5} ; s_{1}, s_{2}, s_{3}, s_{4}, t_{5}\right)\right)$ are different from 0 . This concludes the proof.

Lemma 6.1. Let $F$ be defined as in (6.7) and $x<y$. Then, for any fixed $x$ we have

$$
\lim _{y \rightarrow x} F(x, y)=0
$$

and $F$ is a positive, monotone increasing function of $y$.

Proof. Using L'Hôpital's rule and taking into account that the weights are positive, we get

$$
\lim _{y \rightarrow x} F(x, y)=\lim _{y \rightarrow x} \frac{w_{2}(y) \int_{x}^{y} w_{1}(u) \mathrm{d} u}{w_{2}(y)}=0 .
$$

Moreover, for $x<y$, it is clear that $F(x, y)>0$ and

$$
\begin{aligned}
\frac{\mathrm{d}}{\mathrm{d} y} F(x, y) & =\frac{w_{2}(y) \int_{x}^{y} w_{1}(u) \mathrm{d} u \int_{x}^{y} w_{2}(v) \mathrm{d} v-w_{2}(y) \int_{x}^{y} w_{2}(v) \int_{x}^{v} w_{1}(u) \mathrm{d} u \mathrm{~d} v}{\left(\int_{x}^{y} w_{2}(v) \mathrm{d} v\right)^{2}} \\
& =\frac{w_{2}(y) \int_{x}^{y} w_{2}(v) \int_{v}^{y} w_{1}(u) \mathrm{d} u \mathrm{~d} v}{\left(\int_{x}^{y} w_{2}(v) \mathrm{d} v\right)^{2}}>0 .
\end{aligned}
$$

In view of (6.1), from Proposition 6.2 it follows that the T-mesh in Figure 1 is an example of unstable T-mesh for $C^{1}$ Tchebycheffian spline spaces with $\boldsymbol{T}=\left(\mathbb{T}_{2}, \mathbb{T}_{2}\right)$ and $\mathbb{T}_{2}$ is any ECT-space of dimension 3.

Taking into account Example 5.1, the results of this section also show that the bounds for the dimension of extended complete Tchebycheffian spline spaces provided in Theorem 5.1 are sharp.

\section{Conclusions}

By exploiting the properties of ECT-spaces, in particular the generalized power functions, we have shown that any ECT-space possesses the $\boldsymbol{d}$-sum property. This proves a conjecture stated in [3] and allows us to apply the dimension upper bounds obtained in [16] to any extended complete Tchebycheffian spline space over a planar T-mesh. As a side result, we have provided a link between the $\boldsymbol{d}$-sum property and the minimal support property of Tchebycheffian B-splines.

ECT-spaces are of particular interest as they contain the most attractive Tchebycheff spaces from the application point of view, including trigonometric and hyperbolic spaces. Moreover, the provided upper bounds lead to explicit expressions for the dimension of extended complete Tchebycheffian spline spaces on several relevant T-mesh configurations (where upper and lower bounds agree). This also opens the door for a full generalization to the Tchebycheffian setting of the construction of LR-splines by providing a proper tool to analyze their linear independence.

Furthermore, we have analyzed instability in the dimension of extended complete Tchebycheffian spline spaces over T-meshes, and we have shown that there exist T-meshes such that the corresponding $C^{1}$ spline spaces have unstable dimension for any underlying ECT-space of dimension 3. This gives a further evidence of the total similarity between ECT-spaces and polynomial spaces. 


\section{Acknowledgements}

This work was partially supported by INdAM-GNCS Gruppo Nazionale per il Calcolo Scientifico, by the MIUR "Futuro in Ricerca 2013" program through the project DREAMS, and by the "Mission Sustainability 2017" program of the University of Rome "Tor Vergata" through the project IDEAS. We are also grateful to CIRM - Luminy for the Research-in-Pairs support.

[1] D. Berdinsky, M.-J. Oh, T.-W. Kim, and B. Mourrain. On the problem of instability in the dimension of a spline space over a T-mesh. Comput. Graph. 36 (2012), 507-513.

[2] C. Bracco, T. Lyche, C. Manni, F. Roman, and H. Speleers. Generalized spline spaces over Tmeshes: Dimension formula and locally refined generalized B-splines. Appl. Math. Comput. 272 (2016), 187-198.

[3] C. Bracco, T. Lyche, C. Manni, F. Roman, and H. Speleers. On the dimension of Tchebycheffian spline spaces over planar T-meshes. Comput. Aided Geom. Design 45 (2016), 151-173.

[4] C. Bracco and F. Roman. Spaces of generalized splines over T-meshes. J. Comput. Appl. Math. 294 (2016), 102-123.

[5] J. Deng, F. Chen, and Y. Feng. Dimensions of spline spaces over T-meshes. J. Comput. Appl. Math. 194 (2006), 267-283.

[6] T. Dokken, T. Lyche, and K.F. Pettersen. Polynomial splines over locally refined box-partitions. Comput. Aided Geom. Design 30 (2013), 331-356.

[7] D.R. Forsey and R.H. Bartels. Hierarchical B-spline refinement. Comput. Graph. 22 (1988), 205212 .

[8] C. Giannelli, B. Jüttler, and H. Speleers. THB-splines: The truncated basis for hierarchical splines. Comput. Aided Geom. Design 29 (2012), 485-498.

[9] X. Li and F. Chen. On the instability in the dimension of spline spaces over T-meshes. Comput. Aided Geom. Design 28 (2011), 420-426.

[10] X. Li and J. Deng. On the dimension of spline spaces over T-meshes with smoothing cofactorconformality method. Comput. Aided Geom. Design 41 (2016), 76-86.

[11] X. Li and M.A. Scott. Analysis-suitable T-splines: Characterization, refineability, and approximation. Math. Mod. Meth. Appl. Sci. 24 (2014), 1141-1164.

[12] T. Lyche. A recurrence relation for Chebyshevian B-splines. Constr. Approx. 1 (1985), 155-173.

[13] C. Manni, F. Pelosi, and H. Speleers. Local hierarchical $h$-refinements in IgA based on generalized B-splines. In: M. Floater et al. (eds.), Mathematical Methods for Curves and Surfaces 2012, LNCS 8177 (2014), 341-363.

[14] C. Manni, F. Roman, and H. Speleers. Generalized B-splines in isogeometric analysis. In: G.E. Fasshauer and L.L. Schumaker (eds.), Approximation Theory XV: San Antonio 2016, Springer Proc. Math. Stat. 201 (2017), 239-267.

[15] M.L. Mazure. How to build all Chebyshevian spline spaces good for geometric design? Numer. Math. 119 (2011), 517-556.

[16] B. Mourrain. On the dimension of spline spaces on planar T-meshes. Math. Comp. 83 (2014), $847-871$.

[17] L.L. Schumaker. Spline Functions: Basic Theory, Third Edition. Cambridge University Press (2007).

[18] L.L. Schumaker and L. Wang. Approximation power of polynomial splines on T-meshes. Comput. Aided Geom. Design 29 (2012), 599-612.

[19] T. Sederberg, J. Zheng, A. Bakenov, and A. Nasri. T-splines and T-NURCCs. ACM Trans. Graphics 22 (2003), 477-484. 